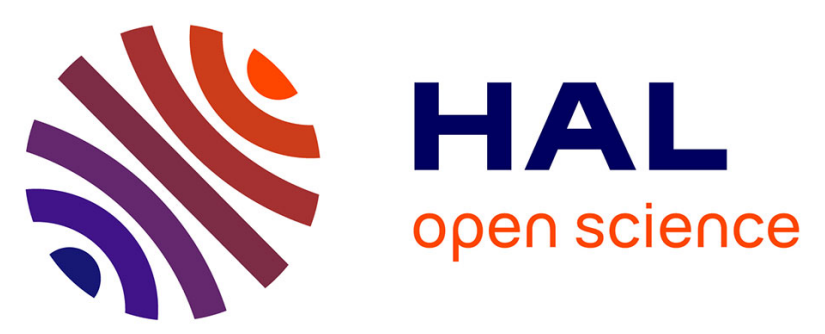

\title{
Two-phase Discrete Fracture Matrix models with linear and nonlinear transmission conditions
}

\author{
Joubine Aghili, Konstantin Brenner, Julian Hennicker, Roland Masson, \\ Laurent Trenty
}

\section{- To cite this version: \\ Joubine Aghili, Konstantin Brenner, Julian Hennicker, Roland Masson, Laurent Trenty. Two-phase Discrete Fracture Matrix models with linear and nonlinear transmission conditions. GEM - Interna- tional Journal on Geomathematics, 2019, 10.1007/s13137-019-0118-6 . hal-01764432v3}

\section{HAL Id: hal-01764432 \\ https://hal.science/hal-01764432v3}

Submitted on 1 Oct 2018

HAL is a multi-disciplinary open access archive for the deposit and dissemination of scientific research documents, whether they are published or not. The documents may come from teaching and research institutions in France or abroad, or from public or private research centers.
L'archive ouverte pluridisciplinaire HAL, est destinée au dépôt et à la diffusion de documents scientifiques de niveau recherche, publiés ou non, émanant des établissements d'enseignement et de recherche français ou étrangers, des laboratoires publics ou privés. 


\title{
Two-phase Discrete Fracture Matrix models with linear and nonlinear transmission conditions
}

\author{
Joubine Aghili ${ }^{1,2}$, Konstantin Brenner ${ }^{1,2}$, Julian Hennicker ${ }^{3}$, Roland Masson ${ }^{1,2}$, and Laurent \\ Trenty $^{4}$ \\ ${ }^{1}$ Team COFFEE, Inria Sophia-Antipolis - Méditerranée, Valbonne, France \\ ${ }^{2}$ Université Côte d'Azur, CNRS, Laboratoire J.A. Dieudonné, Nice, France \\ ${ }^{3}$ Université de Genève, Switzerland \\ ${ }^{4}$ Andra, Chatenay-Malabry, France
}

\begin{abstract}
This work deals with two-phase Discrete Fracture Matrix models coupling the two-phase Darcy flow in the matrix domain to the two-phase Darcy flow in the network of fractures represented as co-dimension one surfaces. Two classes of such hybrid-dimensional models are investigated either based on nonlinear or linear transmission conditions at the matrix-fracture interfaces. The linear transmission conditions include the cell-centred upwind approximation of the phase mobilities classically used in the porous media flow community as well as a basic extension of the continuous phase pressure model accounting for fractures acting as drains. The nonlinear transmission conditions at the matrix-fracture interfaces are based on the normal flux continuity equation for each phase using additional interface phase pressure unknowns. They are compared both in terms of accuracy and numerical efficiency to a reference equi-dimensional model for which the fractures are represented as full-dimensional subdomains. The discretization focuses on Finite Volume cellcentred Two-Point Flux Approximation which is combined with a local nonlinear solver allowing to eliminate efficiently the additional matrix-fracture interfacial unknowns together with the nonlinear transmission conditions. 2D numerical experiments illustrate the better accuracy provided by the nonlinear transmission conditions compared to their linear approximations with a moderate computational overhead obtained thanks to the local nonlinear elimination at the matrix-fracture interfaces. The numerical section is complemented by a comparison of the reduced models on a 3D test case using the Vertex Approximate Gradient scheme.
\end{abstract}

\section{Introduction}

This work deals with numerical modeling of two-phase flow in fractured porous media, for which the fracture network is represented as a manifold of co-dimension one with respect to matrix domain. This approach gives rise to so-called Discrete Fracture Matrix (DFM) models also termed hybriddimensional models, which differ among themselves mostly by the interface condition imposed at matrix-fracture $(m f)$ interfaces. Various interface conditions have been proposed in the literature, both for single-phase and multi-phase flows. For single-phase flows there are two major approaches - the first, designed for modeling highly conductive fractures and referred to as continuous pressure model $[2,10]$, assumes the continuity of the fluid pressure at the $m f$ interface; the second approach, referred to as discontinuous pressure model $[16,19,5,8]$, allows to represent fractures acting as permeability barriers by imposing Robin-type condition at $m f$ interface.

When the modeling of multi-phase flow is concerned, three major types of models may be distinguished. The first and most common type is based on the straightforward adaptation of single-phase

E-mail of corresponding author: konstantin.brenner@unice.fr 
continuous pressure model to the multi-phase setting (see [7, 25, 23, 18, 12, 11]), it assumes the continuity of each phase pressure at $m f$ interfaces. As for single-phase flow this approach can not account for fractures acting as barriers.

Another existing type of models, accounting for both drains or barriers, amounts to eliminate the interfacial phase pressures using, for each phase, the linear single-phase Darcy flux conservation equation at the $m f$ interfaces. It is usually combined with Two-Point [21] or MultiPoint $[28,27,4]$ cell-centred finite volume schemes for which the interfacial unknowns can be easily eliminated when building the single phase Darcy flux transmissibilities. Both this type of model and the previous continuous pressure model are termed in the following hybrid-dimensional $m f$ linear models in the sense that they are based on linear transmission conditions for the phase pressures.

Finally a few works have considered nonlinear interface conditions, namely $[9,14]$ using a formulation based on phase pressure and upwinding of the mobilities, and [20,3] using a global pressure formulation. Such nonlinear interface conditions account for the permeability jump as well as for the discontinuity of phase mobility at $m f$ interface. This type of model is termed in the following hybrid-dimensional $m f$ nonlinear model since it is based on the nonlinear flux continuity equations at the $m f$ interface.

This article is a follow-up of the work presented in [9] on the comparison of the hybrid-dimensional $m f$ nonlinear and linear models using a reference equi-dimensional solution for which the fractures are represented as full-dimensional subdomains. The work presented in [9] is limited to rather large apertures due to the difficulty to obtain reference equi-dimensional solutions using nodal based discretizations like the Vertex Approximate Gradient (VAG) scheme. The present work focuses on cellcentred discretizations with Two-Point Flux Approximations (TPFA) on orthogonal meshes in order to obtain reference equi-dimensional solutions for small apertures. We will show in particular that, in contrast with single-phase flow (see [17]), the continuous pressure model may fail even when fractures are highly conductive. This typically happens when the fracture network is dry, and hence, due to the low relative permeability the wetting phase pressure is no longer continuous across the fracture. We also investigate the effect of gravitational phase segregation within the fracture network, which can only be captured by the nonlinear $m f$ model. We also compare the efficiency of the models in terms of linear and nonlinear convergence as well as in terms of overall CPU time. The TPFA discretization of the hybrid-dimensional $m f$ nonlinear model is combined with a new nonlinear interface solver in order to eliminate the $m f$ interface unknowns. This strategy allows to obtain a better approximation at a moderate additional cost compared with the $m f$ linear models.

The remainder of the present article is organized as follows. In Section 2 we recall the hybriddimensional $m f$ nonlinear two-phase flow model from [9]. In Section 3, the cell-centred TPFA discretization of this model is introduced as well as the TPFA discretizations of the $m f$ linear two-phase flow models based on linear $m f$ interface conditions. In Section 4 the local nonlinear interface problem is studied and an algorithm for finding its solutions is presented. In Section 5, numerical comparisons on $2 \mathrm{D}$ test cases of the hybrid dimensional $m f$ nonlinear and linear models are considered using equidimensional reference solutions. Section 6 presents the comparison of the models for a 3D test case using the Vertex Approximate Gradient scheme introduced in [9] for hybrid-dimensional two phase Darcy flow models.

\section{Continuous model}

Let $\Omega$ be an open bounded domain of $\mathbb{R}^{d}, d=2,3$ assumed to be polyhedral for $d=3$ (and polygonal for $d=2$ ). To fix ideas the dimension will be fixed to $d=3$ when it needs to be specified, for instance in the naming of the geometrical objects or for the space discretization in the next section. The adaptations to the case $d=2$ are straightforward. Let $\bar{\Gamma}=\bigcup_{i \in I} \bar{\Gamma}_{i}$ denotes the network of fractures $\Gamma_{i} \subset \Omega, i \in I$, such that each $\Gamma_{i}$ is a planar polygonal simply connected open domain included in some plane of $\mathbb{R}^{d}$. Without restriction of generality, we will assume that the fractures may intersect exclusively at their boundaries (see Figure 1), that is for any $i, j \in I, i \neq j$ one has $\Gamma_{i} \cap \Gamma_{j}=\emptyset$, but 
not necessarily $\bar{\Gamma}_{i} \cap \bar{\Gamma}_{j}=\emptyset$.

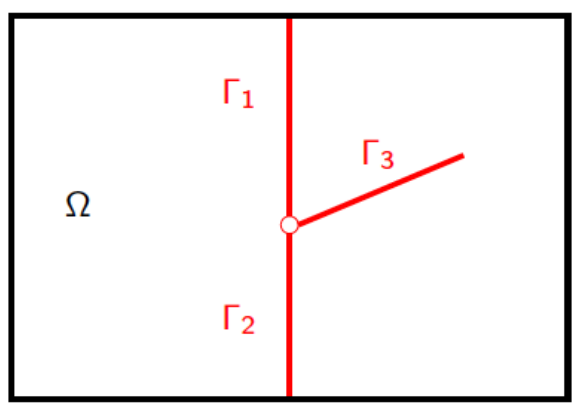

Figure 1: Example of a 2D domain $\Omega$ with 3 intersecting fractures $\Gamma_{i}, i=1,2,3$.

In the matrix domain $\Omega$ (resp. in the fracture network $\Gamma$ ), we denote by $\phi_{m}(\mathbf{x})\left(\operatorname{resp} . \phi_{f}(\mathbf{x})\right)$ the porosity, by $\Lambda_{m}(\mathbf{x})$ (resp. $\Lambda_{f}(\mathbf{x})$ ) the permeability (resp. tangential permeability) tensor. The permeability tensor in the fracture network is assumed to have the fracture normal vector as principal direction and $\lambda_{f, n}(\mathbf{x})$ denotes the corresponding fracture normal permeability. The thickness of the fractures is denoted by $d_{f}(\mathbf{x})$ for $\mathbf{x} \in \Gamma$.

For each phase $\alpha=w, n w$ (where $w$ stands for "wetting" and $n w$ for "non-wetting" phases) we denote by $k_{r, m}^{\alpha}(s)\left(\right.$ resp. $\left.k_{r, f}^{\alpha}(s)\right)$, the phase relative permeabilities and by $S_{m}^{\alpha}(\pi)\left(\operatorname{resp} . S_{f}^{\alpha}(\pi)\right)$ the capillary pressure-saturation curves. For simplicity and since we focus on matrix-fracture network interfaces, its is assumed that both the matrix and fracture domains are homogeneous with respect to capillary pressure and relative permeability laws. We will assume that for each $j=m, f$ the capillary pressure-saturation and relative permeability curves satisfy

- $S_{j}^{n w}$ is a non-decreasing continuous function from $\mathbb{R}$ onto $[0,1)$ such that there exist the so-called entry pressure $\pi_{j, e}$ satisfying $S_{j}^{n w}\left(\pi \leq \pi_{j, e}\right)=0$. The function $S_{j}^{w}$ satisfy $S_{j}^{w}(\pi)=1-S_{j}^{n w}(\pi)$ for all $\pi \in \mathbb{R}$.

- $k_{r, j}^{\alpha}$, for each $\alpha=w, n w$, is a non-decreasing continuous function on $[0,1]$ such that $k_{r, j}^{\alpha}(0)=0$ and $k_{r, j}^{\alpha}(1)>0$.

Typical examples of such relations are given by Brooks-Corey [13], Bentsen-Anli [6] or Van GenuchtenMualem [24, 29] laws.

For $\alpha=w, n w$, we will denote by $\rho^{\alpha}$ the phase densities and by $\mu^{\alpha}$ the phase dynamic viscosities, which for the sake of clarity, are assumed constant. The mobilities of the phases (ratio of the phase relative permeability to the phase viscosity) will be denoted by $k_{m}^{\alpha}$ and $k_{f}^{\alpha}$. We denote by $p_{m}^{\alpha}$ (resp. $p_{f}^{\alpha}$ ) the pressure of phase $\alpha=w, n w$ and by $s_{m}^{\alpha}$ (resp. $s_{f}^{\alpha}$ ) the saturation of phase $\alpha=w, n w$ in the matrix (resp. the fracture network) domain.

Matrix equations. The Darcy flux of phase $\alpha=w, n w$ in the matrix domain is defined by

$$
\mathbf{q}_{m}^{\alpha}=-k_{m}^{\alpha}\left(s_{m}^{\alpha}\right) \Lambda_{m}\left(\nabla p_{m}^{\alpha}-\rho^{\alpha} \mathbf{g}\right),
$$

where $\mathbf{g}=-g \nabla z$ stands for the gravity vector. The flow in the matrix domain is described for both phases $\alpha=w, n w$ by the volume balance equation

$$
\phi_{m} \partial_{t} s_{m}^{\alpha}+\operatorname{div}\left(\mathbf{q}_{m}^{\alpha}\right)=0,
$$

and the macroscopic capillary pressure law

$$
s_{m}^{\alpha}=S_{m}^{\alpha}\left(p_{m}^{n w}-p_{m}^{w}\right) .
$$

Fracture network equations. For each fracture $\Gamma_{i}$ we identify the two sides + and - of $\Gamma_{i}$ in $\Omega \backslash \bar{\Gamma}$. We formally denote by $\gamma_{i}^{+}$and $\gamma_{i}^{-}$the corresponding trace operators. The unit normal vectors at $\Gamma_{i}$ 
outward to the side $+\left(\right.$ resp. - ) are denoted by $\mathbf{n}_{i}^{+}\left(\right.$resp. $\left.\mathbf{n}_{i}^{-}\right)$, and $\mathbf{q}_{m}^{\alpha,+} \cdot \mathbf{n}_{i}^{+}\left(\operatorname{resp} . \mathbf{q}_{m}^{\alpha,-} \cdot \mathbf{n}_{i}^{-}\right)$formally denotes the normal trace of the matrix fluxes at the + side (resp. - side) of the fracture $\Gamma_{i}$ oriented outward to the matrix. In addition, for $i \in I$, we denote by $\nabla_{\tau_{i}}$ the tangential gradient, and by $\operatorname{div}_{\tau_{i}}$ the tangential divergence operators.

The Darcy flux of phase $\alpha=w, n w$ in the fracture $\Gamma_{i}$ integrated over the width of the fracture is defined by

$$
\mathbf{q}_{f, i}^{\alpha}=-d_{f} k_{f}^{\alpha}\left(s_{f}^{\alpha}\right) \Lambda_{f}\left(\nabla_{\tau_{i}} p_{f}^{\alpha}-\rho^{\alpha} \mathbf{g}_{\tau_{i}}\right),
$$

with $\mathbf{g}_{\tau_{i}}=\mathbf{g}-\left(\mathbf{g} \cdot \mathbf{n}_{i}^{+}\right) \mathbf{n}_{i}^{+}$. The flow in each fracture $\Gamma_{i}$ is described for both phases $\alpha=w, n w$ by

$$
d_{f} \phi_{f} \partial_{t} s_{f}^{\alpha}+\operatorname{div}_{\tau_{i}}\left(\mathbf{q}_{f, i}^{\alpha}\right)-\mathbf{q}_{m}^{\alpha,+} \cdot \mathbf{n}_{i}^{+}-\mathbf{q}_{m}^{\alpha,-} \cdot \mathbf{n}_{i}^{-}=0,
$$

and

$$
s_{f}^{\alpha}=S_{f}^{\alpha}\left(p_{f}^{n w}-p_{f}^{w}\right) .
$$

Matrix-fracture interface conditions. The matrix and the fracture equations are coupled by Robin boundary conditions which make use of two-point approximations of the normal fluxes within the fracture. Let us first define for $i \in I$ and for both phases $\alpha=w, n w$ the single phase normal fluxes in the fracture

$$
V_{f, i, n}^{\alpha, \pm}=\lambda_{f, n}\left(\frac{\gamma_{i}^{ \pm} p_{m}^{\alpha}-p_{f}^{\alpha}}{d_{f} / 2}-\rho^{\alpha} \mathbf{g} \cdot \mathbf{n}_{i}^{ \pm}\right) .
$$

For any $a \in \mathbb{R}$, let us set $a^{+}=\max \{0, a\}$ and $a^{-}=-(-a)^{+}$, the condition coupling matrix and fracture unknowns then reads

$$
\mathbf{q}_{m}^{\alpha, \pm} \cdot \mathbf{n}_{i}^{ \pm}=k_{f}^{\alpha}\left(S_{f}^{\alpha}\left(\gamma_{i}^{ \pm} p_{m}^{n w}-\gamma_{i}^{ \pm} p_{m}^{w}\right)\right)\left(V_{f, i, n}^{\alpha, \pm}\right)^{+}+k_{f}^{\alpha}\left(s_{f}^{\alpha}\right)\left(V_{f, i, n}^{\alpha, \pm}\right)^{-} .
$$

The hybrid dimensional two-phase flow model looks for $\left(p_{m}^{\alpha}, p_{f}^{\alpha}\right)_{\alpha=w, n w}$ satisfying (1)-(8). In addition to (1)-(8) we prescribe no-flux boundary conditions at the tips of the immersed fractures, that is to say on $\partial \Gamma \backslash \partial \Omega$, and the flux conservation and pressure continuity conditions at the fracture intersections. We refer to [12] for a more detailed presentation of those conditions. Finally, one should provide some appropriate initial and boundary data.

\section{Discrete models}

The approximation of the diffusive fluxes relies on Two-Point Flux Approximation. This approximation is consistent if the permeability tensor is isotropic and if the mesh satisfies the so-called orthogonality condition (see e.g. [15]). Below we introduce some notations related to the space discretization, for the sake of clarity we present the discretization for $d=3$, the extension to $d=2$ is straightforward.

\subsection{Space discretization}

We will denote by $\mathcal{M}$ the set of disjoint open polyhedral cells, by $\mathcal{F}$ the set of faces and by $\mathcal{E}$ the set of edges of the mesh. For each cell $K \in \mathcal{M}$, we denote by $\mathcal{F}_{K} \subset \mathcal{F}$ the set of its faces and for each face $\sigma \in \mathcal{F}$, we denote by $\mathcal{E}_{\sigma}$ the set of its edges. In addition, the set of cells sharing a given face $\sigma \in \mathcal{F}$ is denoted by $\mathcal{M}_{\sigma}$, and the set of faces sharing a given edge $e \in \mathcal{E}$ is denoted by $\mathcal{F}_{e}$.

The mesh is supposed to be conforming w.r.t. the fracture network $\bar{\Gamma}$ in the sense that, for each $i \in I$, there exists a subset $\mathcal{F}_{\Gamma_{i}}$ of $\mathcal{F}$ such that $\bar{\Gamma}_{i}=\bigcup_{\sigma \in \mathcal{F}_{\Gamma_{i}}} \bar{\sigma}$. We will denote by $\mathcal{F}_{\Gamma}$ the subset $\bigcup_{i \in I} \mathcal{F}_{\Gamma_{i}}$ of fracture faces.

For all $K \in \mathcal{M}$ and $\sigma \in \mathcal{F}_{K}$, we denote by $\mathbf{n}_{K \sigma}$ the unit outward normal vector to $\sigma$ w.r.t $K$, and similarly, for all $\sigma \in \mathcal{F}_{\Gamma}$ and $e \in \mathcal{E}$, we denote by $\mathbf{n}_{\sigma e}$ the unit outward normal vector to $e$ w.r.t $\sigma$. Finally, we denote by $|\sigma|, \sigma \in \mathcal{F}$ the $d-1$ dimensional measure of $\sigma$ and by $|e|, e \in \mathcal{E}$ the $d-2$ dimensional measure of $e$. 
The degrees of freedom of the discretization scheme are associated with the "cell centres" $\mathbf{x}_{K} \in K$, the "centres" of the fracture faces $\mathbf{x}_{\sigma} \in \sigma$, and the "centres" $\mathbf{x}_{e} \in e$ of certain edges of the mesh. More precisely, in order to deal with the intersection of multiple fractures at a given edge $e \in \mathcal{E}$ we associate to it a degree of freedom and a certain porous volume. We will denote by $\mathcal{E}_{\star}$ the subset of $\mathcal{E}$ such that for all $e \in \mathcal{E}_{\star}$ the set $\mathcal{F}_{\Gamma} \cap \mathcal{F}_{e}$ has at least 3 elements. The space of degrees of freedom is defined by

$$
X_{\mathcal{D}}=\left\{p_{K}, p_{\sigma}, p_{e} \in \mathbb{R}, K \in \mathcal{M}, \sigma \in \mathcal{F}_{\Gamma}, e \in \mathcal{E}_{\star}\right\} .
$$

Orthogonality condition. Let us denote by $\mathbf{x}_{K \sigma}$ the projection of $\mathbf{x}_{K}$ on the face $\sigma \in \mathcal{F}_{K}$ and by $\mathbf{x}_{\sigma e}$ the projection of $\mathbf{x}_{\sigma}$ on the edge $e \in \mathcal{E}_{\sigma}$. We assume that the mesh satisfies the following conditions

- for any interior face $\sigma$ and $K, L \in \mathcal{M}$ such that $\sigma=\mathcal{F}_{K} \cap \mathcal{F}_{L}$ one has $\mathbf{x}_{K \sigma}=\mathbf{x}_{L \sigma}$,

- for any fracture face $\sigma \in \mathcal{F}_{\Gamma}$ and $K$ such that $\sigma \in \mathcal{F}_{K}$ one has $\mathbf{x}_{K \sigma}=\mathbf{x}_{\sigma}$,

and

- for any interior fracture edge $e$ and $\sigma, \sigma^{\prime} \in \mathcal{F}_{\Gamma}$ such that $e=\mathcal{E}_{\sigma} \cap \mathcal{E}_{\sigma^{\prime}}$ one has $\mathbf{x}_{\sigma e}=\mathbf{x}_{\sigma^{\prime} e}$,

- for any intersection edge $e \in \mathcal{E}_{\star}$ and $\sigma \in \mathcal{F}_{\Gamma}$ such that $e \in \mathcal{E}_{\sigma}$ one has $\mathbf{x}_{\sigma e}=\mathbf{x}_{e}$.

In addition we assume that the porosity $\phi_{m}$ (resp. $\phi_{f}$ ) is cell-wise (resp. face-wise) constant, and that the permeability tensor $\Lambda_{m}$ (resp. $\Lambda_{f}$ ) is cell-wise (resp. face-wise) constant and isotropic.

\subsection{Discrete equations}

For $N \in \mathbb{N}^{*}$, let us consider the discretization $t^{0}=0<t^{1}<\cdots<t^{n-1}<t^{n}<\cdots<t^{N}=T$ of the time interval $[0, T]$. We denote the time steps by $\Delta t^{n}=t^{n}-t^{n-1}$ for all $n=1, \cdots, N$. For $K \in \mathcal{M}$, $\sigma \in \mathcal{F}_{K}$ and $\alpha=w, n w$, we denote by $F_{K \sigma}^{\alpha, n}$ the approximation of the Darcy flux through the face $\sigma$ of the cell $K$

$$
F_{K \sigma}^{\alpha, n} \approx \frac{1}{\Delta t^{n}} \int_{t_{n-1}}^{t_{n}} \int_{\sigma} \Lambda_{m}\left(\mathbf{x}_{K}\right) k_{m}^{\alpha}\left(s_{m}^{\alpha}\right)\left(\nabla p_{m}^{\alpha}-\rho^{\alpha} \mathbf{g}\right) \cdot \mathbf{n}_{K \sigma} d \sigma(\mathbf{x}) d t .
$$

Similarly, for $\sigma \in \mathcal{F}_{\Gamma_{i}}, i \in I, e \in \mathcal{E}_{\sigma}$ and $\alpha=w, n w$, we denote by $F_{\sigma e}^{\alpha, n}$ the approximation of the Darcy flux through the edge $e$ of the fracture face $\sigma$

$$
F_{\sigma e}^{\alpha, n} \approx \frac{1}{\Delta t^{n}} \int_{t_{n-1}}^{t_{n}} \int_{e} d_{f}(\mathbf{x}) \Lambda_{f}\left(\mathbf{x}_{\sigma}\right) k_{f}^{\alpha}\left(s_{f}^{\alpha}\right)\left(\nabla_{\tau_{i}} p_{f}^{\alpha}-\rho^{\alpha} \mathbf{g}_{\tau_{i}}\right) \cdot \mathbf{n}_{\sigma e} d e(\mathbf{x}) d t .
$$

Those discrete fluxes are going to be defined later in this section. Let us first present the general layout of the numerical scheme, for this purpose we define the porous volume associated with the degrees of freedom. For all $K \in \mathcal{M}$ we simply set $\phi_{K}=\int_{K} \phi_{m}(\mathbf{x}) d \mathbf{x}$, while the fracture porous volume has to be distributed between the degrees of freedom associated with fracture faces $\sigma \in \mathcal{F}_{\Gamma}$ and intersection edges $e \in \mathcal{E}_{\star}$. For each $\sigma \in \mathcal{F}_{\Gamma}$ such that $\mathcal{E}_{\sigma} \cap \mathcal{E}_{\star} \neq \emptyset$ we define a set of non-negative volume fractions $\left(\alpha_{\sigma e}\right)_{e \in \mathcal{E}_{\sigma} \cap \mathcal{E}_{\star}}$ satisfying $\sum_{e \in \mathcal{E}_{\sigma} \cap \mathcal{E}_{\star}} \alpha_{\sigma e}<1$, and we set

$$
\phi_{\sigma}=\left(1-\sum_{e \in \mathcal{E}_{\sigma} \cap \mathcal{E}_{\star}} \alpha_{\sigma e}\right) \int_{\sigma} d_{f}(\mathbf{x}) \phi_{f}(\mathbf{x}) d \sigma(\mathbf{x}),
$$

and

$$
\phi_{e}=\sum_{\sigma \in \mathcal{F}_{e} \cap \mathcal{F}_{\Gamma}} \alpha_{\sigma e} \int_{\sigma} d_{f}(\mathbf{x}) \phi_{f}(\mathbf{x}) d \sigma(\mathbf{x}) .
$$

For each $\sigma \in \mathcal{F}_{\Gamma}$ such that $\mathcal{E}_{\sigma} \cap \mathcal{E}_{\star}=\emptyset$, we simply set $\phi_{\sigma}=\int_{\sigma} d_{f}(\mathbf{x}) \phi_{f}(\mathbf{x}) d \sigma(\mathbf{x})$. Note that the above conservative distribution of a porous volume from adjacent fracture faces to their intersecting edge 
$e \in \mathcal{E}_{\star}$ is a simple and efficient way to circumvent the classical bad conditioning issue induced by fracture intersections. Alternative approaches based on the Star Delta [21] transformation or a Schur complement eliminating the edge $e \in \mathcal{E}_{\star}$ unknowns [27] are basically limited to linear problems.

The finite volume scheme looks for $p^{\alpha, n} \in X_{\mathcal{D}}, \alpha=w, n w, n=1, \ldots, N$ such that, for $\alpha=w, n w$, the following set of equations is satisfied:

$$
\frac{\phi_{K}}{\Delta t^{n}}\left(s_{K}^{\alpha, n}-s_{K}^{\alpha, n-1}\right)+\sum_{\sigma \in \mathcal{F}_{K} \backslash \mathcal{F}_{\Gamma}} F_{K \sigma}^{\alpha, n}+\sum_{\sigma \in \mathcal{F}_{K} \cap \mathcal{F}_{\Gamma}} F_{K \sigma}^{\alpha, n}=0
$$

for all $K \in \mathcal{M}$, and

$$
\frac{\phi_{\sigma}}{\Delta t^{n}}\left(s_{\sigma}^{\alpha, n}-s_{\sigma}^{\alpha, n-1}\right)+\sum_{e \in \mathcal{E}_{\sigma} \backslash \mathcal{E}_{\star}} F_{\sigma e}^{\alpha, n}+\sum_{e \in \mathcal{E}_{\sigma} \cap \mathcal{E}_{\star}} F_{\sigma e}^{\alpha, n}-\sum_{K \in \mathcal{M}_{\sigma}} F_{K \sigma}^{\alpha, n}=0,
$$

for all $\sigma \in \mathcal{F}_{\Gamma}$, and

$$
\frac{\phi_{e}}{\Delta t^{n}}\left(s_{e}^{\alpha, n}-s_{e}^{\alpha, n-1}\right)-\sum_{\sigma \in \mathcal{F}_{e} \cap \mathcal{F}_{\Gamma}} F_{\sigma e}^{\alpha, n}=0
$$

for all $e \in \mathcal{E}_{\star}$, with

$$
\begin{gathered}
s_{K}^{\alpha, n}=S_{m}^{\alpha}\left(p_{K}^{n w, n}-p_{K}^{w, n}\right) \quad \text { for all } K \in \mathcal{M}, \\
s_{\nu}^{\alpha, n}=S_{f}^{\alpha}\left(p_{\nu}^{n w, n}-p_{\nu}^{w, n}\right) \quad \text { for all } \nu \in \mathcal{F}_{\Gamma} \cup \mathcal{E}_{\star},
\end{gathered}
$$

where $p_{\nu}^{\alpha, n}$ denotes the approximation of the matrix (resp. fracture) phase pressure at point $\mathbf{x}_{\nu}$ for $\nu \in \mathcal{M}$ (resp. $\nu \in \mathcal{F}_{\Gamma} \cup \mathcal{E}_{\star}$ ).

\subsection{Discrete fluxes}

In this subsection, we give the definition of the discrete fluxes using the following additional notations. For all degrees of freedom $\nu \in \mathcal{M} \cup \mathcal{F}_{\Gamma} \cup \mathcal{E}_{\star}$ and $n=1, \ldots, N$ we denote by $w_{\nu}^{\alpha, n}$ the approximation of the phase potential at point $\mathbf{x}_{\nu}$ defined by

$$
w_{\nu}^{\alpha, n}=p_{\nu}^{\alpha, n}+\rho^{\alpha} g z_{\nu} .
$$

Matrix-matrix fluxes. Let us consider an interface $\sigma$ between two neighboring cells $K, L \in \mathcal{M}$ such that $\sigma=\mathcal{F}_{K} \cap \mathcal{F}_{L} \neq \emptyset$ and $\sigma \notin \mathcal{F}_{\Gamma}$. Let us define the half-transmissibilities defined for all $K \in \mathcal{M}$ and $\sigma \in \mathcal{F}_{K}$ by

$$
T_{K \sigma}=\Lambda_{m}\left(\mathbf{x}_{K}\right) \frac{|\sigma|}{\left|\mathbf{x}_{K}-\mathbf{x}_{\sigma}\right|} .
$$

Let $k_{K \sigma}^{\alpha, n}$ and $w_{\sigma}^{\alpha, n}$ be some approximate values of the phase mobility and of the phase potential at the interface $\sigma$. Then, we set

$$
V_{K \sigma}^{\alpha, n}=T_{K \sigma}\left(w_{K}^{\alpha, n}-w_{\sigma}^{\alpha, n}\right) \quad \text { and } \quad F_{K \sigma}^{\alpha, n}=k_{K \sigma}^{\alpha, n} V_{K \sigma}^{\alpha, n},
$$

and flux continuity is imposed at the interface

$$
F_{K \sigma}^{\alpha, n}+F_{L \sigma}^{\alpha, n}=0
$$

Since, except for the $m f$ interfaces, the capillary pressure-saturation and relative permeability curves do not depend on the space variable, the saturation jump at the interface $\sigma$ can be neglected and it is natural to assume that $k_{K \sigma}^{\alpha, n}=k_{L \sigma}^{\alpha, n}$. Hence equation (12) reduces to

$$
V_{K \sigma}^{\alpha, n}+V_{L \sigma}^{\alpha, n}=0
$$

and the interface potential $w_{\sigma}^{\alpha, n}$ can be linearly eliminated from (13) yielding

$$
V_{K \sigma}^{\alpha, n}=\frac{T_{K \sigma} T_{L \sigma}}{T_{K \sigma}+T_{L \sigma}}\left(w_{K}^{\alpha, n}-w_{L}^{\alpha, n}\right) .
$$


Using the upstream approximation of the relative mobility, we come up with the following definition of the discrete flux

$$
F_{K \sigma}^{\alpha, n}=k_{m}^{\alpha}\left(s_{K}^{\alpha, n}\right)\left(V_{K \sigma}^{\alpha, n}\right)^{+}+k_{m}^{\alpha}\left(s_{L}^{\alpha, n}\right)\left(V_{K \sigma}^{\alpha, n}\right)^{-} .
$$

Fracture-fracture fluxes. Similarly to the previous matrix-matrix fluxes, we define for all $\sigma \in \mathcal{F}_{\Gamma}$ and $e \in \mathcal{E}_{\sigma}$ the half-transmissibilities

$$
T_{\sigma e}=\Lambda_{f}\left(\mathbf{x}_{\sigma}\right) \frac{d_{f}\left(\mathbf{x}_{\sigma}\right)|e|}{\left|\mathbf{x}_{\sigma}-\mathbf{x}_{e}\right|}
$$

Let $\sigma$ and $\sigma^{\prime}$ be a couple of intersecting fracture faces and let $e=\bar{\sigma} \cap \bar{\sigma}^{\prime} \notin \mathcal{E}_{\star}$, the flux between $\sigma$ and $\sigma^{\prime}$ is defined by

$$
F_{\sigma e}^{\alpha, n}=k_{f}^{\alpha}\left(s_{\sigma}^{\alpha, n}\right)\left(V_{\sigma e}^{\alpha, n}\right)^{+}+k_{f}^{\alpha}\left(s_{\sigma^{\prime}}^{\alpha, n}\right)\left(V_{\sigma e}^{\alpha, n}\right)^{-},
$$

with

$$
V_{\sigma e}^{\alpha, n}=\frac{T_{\sigma e} T_{\sigma^{\prime} e}}{T_{\sigma e}+T_{\sigma e}}\left(w_{\sigma}^{\alpha, n}-w_{\sigma^{\prime}}^{\alpha, n}\right) .
$$

Let $\sigma \in \mathcal{F}_{\Gamma}$ and $e \in \mathcal{E}_{\sigma} \cap \mathcal{E}_{\star}$, we set

$$
F_{\sigma e}^{\alpha, n}=k_{f}^{\alpha}\left(s_{\sigma}^{\alpha, n}\right)\left(V_{\sigma e}^{\alpha, n}\right)^{+}+k_{f}^{\alpha}\left(s_{e}^{\alpha, n}\right)\left(V_{\sigma e}^{\alpha, n}\right)^{-},
$$

with

$$
V_{\sigma e}^{\alpha, n}=T_{\sigma e}\left(w_{\sigma}^{\alpha, n}-w_{e}^{\alpha, n}\right) .
$$

Matrix-fracture fluxes with nonlinear transmission conditions. For $K \in \mathcal{M}$ and $\sigma \in \mathcal{F}_{K} \cap \mathcal{F}_{\Gamma}$ let us denote by $w_{K \sigma}^{\alpha, n}$ an approximation of the trace of $\left.w_{m}^{\alpha}\left(\cdot, t_{n}\right)\right|_{K}$ at the interface $\sigma$ on the cell $K$ side (interface $K \sigma$ ) using

$$
z_{K \sigma}=z_{\sigma}+\frac{\left(z_{K}-z_{\sigma}\right) d_{f}\left(\mathbf{x}_{\sigma}\right)}{2\left|\mathbf{x}_{K}-\mathbf{x}_{\sigma}\right|} .
$$

Let us define the phase saturation $s_{K, K \sigma}^{\alpha, n}$ at the interface $K \sigma$ on the matrix side by

$$
s_{K, K \sigma}^{\alpha, n}=S_{m}^{\alpha}\left(w_{K \sigma}^{n w, n}-w_{K \sigma}^{w, n}-\left(\rho^{n w}-\rho^{w}\right) g z_{K \sigma}\right),
$$

and the phase saturation $s_{\sigma, K \sigma}^{\alpha, n}$ at the interface $K \sigma$ on the fracture side by

$$
s_{\sigma, K \sigma}^{\alpha, n}=S_{f}^{\alpha}\left(w_{K \sigma}^{n w, n}-w_{K \sigma}^{w, n}-\left(\rho^{n w}-\rho^{w}\right) g z_{K \sigma}\right) .
$$

The left-hand side of (8) is discretized by

$$
F_{K, K \sigma}^{\alpha, n}=k_{m}^{\alpha}\left(s_{K}^{\alpha, n}\right) T_{K \sigma}\left(w_{K}^{\alpha, n}-w_{K \sigma}^{\alpha, n}\right)^{+}+k_{m}^{\alpha}\left(s_{K, K \sigma}^{\alpha, n}\right) T_{K \sigma}\left(w_{K}^{\alpha, n}-w_{K \sigma}^{\alpha, n}\right)^{-},
$$

while the discretization of the right-hand side of (8) is given by $-F_{\sigma, K \sigma}^{\alpha, n}$ with

$$
F_{\sigma, K \sigma}^{\alpha, n}=k_{f}^{\alpha}\left(s_{\sigma}^{\alpha, n}\right) T_{\sigma}\left(w_{\sigma}^{\alpha, n}-w_{K \sigma}^{\alpha, n}\right)^{+}+k_{f}^{\alpha}\left(s_{\sigma, K \sigma}^{\alpha, n}\right) T_{\sigma}\left(w_{\sigma}^{\alpha, n}-w_{K \sigma}^{\alpha, n}\right)^{-},
$$

with

$$
T_{\sigma}=\lambda_{f, n}\left(\mathbf{x}_{\sigma}\right) \frac{|\sigma|}{d_{f}\left(\mathbf{x}_{\sigma}\right) / 2} .
$$

Gathering (17) and (18) in the flux continuity equation (8) for each phase, we find

$$
F_{K, K \sigma}^{\alpha, n}+F_{\sigma, K \sigma}^{\alpha, n}=0, \quad \alpha=w, n w .
$$

The interface potentials $\left(w_{K \sigma}^{\alpha, n}\right)_{\alpha=w, n w}$ are eliminated by solving the two equations (19) using the algorithm detailed in the next section. Then, the matrix-fracture flux $F_{K \sigma}^{\alpha, n}$ depending only on $p_{\nu}^{\beta}$, $\beta=w, n w, \nu=K, \sigma$ is defined by

$$
F_{K \sigma}^{\alpha, n}=F_{K, K \sigma}^{\alpha, n}=-F_{\sigma, K \sigma}^{\alpha, n}
$$


Matrix-fracture fluxes with linear transmission conditions. The evaluation of the matrixfracture fluxes defined by (17)-(18)-(19) requires the solution of local nonlinear problems which may produce a computational overhead compared to some simpler approaches. Following [9], we consider two alternative definitions of $m f$ discrete fluxes. The first one corresponds to the classical flux approximation proposed in [21]. It assumes, as for matrix-matrix (or fracture-fracture) fluxes, the continuity of the phase mobility at the matrix-fracture interface defined by its upstream value, precisely we define

$$
F_{K \sigma}^{\alpha, n}=k_{m}^{\alpha}\left(s_{K}^{\alpha, n}\right)\left(V_{K \sigma}^{\alpha, n}\right)^{+}+k_{f}^{\alpha}\left(s_{\sigma}^{\alpha, n}\right)\left(V_{K \sigma}^{\alpha, n}\right)^{-},
$$

with

$$
V_{K \sigma}^{\alpha, n}=\frac{T_{K \sigma} T_{\sigma}}{T_{K \sigma}+T_{\sigma}}\left(w_{K}^{\alpha, n}-w_{\sigma}^{\alpha, n}\right) .
$$

The second alternative is inspired by the continuous pressure model proposed in [12], which assumes that the phase pressure is the same within the fracture and at the $m f$ interfaces. This model can be extended to account also for low permeable fractures as follows, although it does not account for capillary barriers as will be checked in the numerical experiments. Precisely, it is defined by the flux

$$
F_{K \sigma}^{\alpha, n}=k_{m}^{\alpha}\left(s_{K}^{\alpha, n}\right)\left(V_{K \sigma}^{\alpha, n}\right)^{+}+k_{m}^{\alpha}\left(s_{K \sigma}^{\alpha, n}\right)\left(V_{K \sigma}^{\alpha, n}\right)^{-},
$$

with $V_{K \sigma}^{\alpha, n}$ given again by (22) and

$$
s_{K \sigma}^{\alpha, n}=S_{m}^{\alpha}\left(w_{\sigma}^{n w, n}-w_{\sigma}^{w, n}-\left(\rho^{n w}-\rho^{w}\right) g z_{\sigma}\right) .
$$

For permeable fractures the flux designed by (23) is essentially equivalent to the one defined in [12].

The model provided by the matrix-fracture fluxes (21) is termed in the following the hybriddimensional $m f$ linear $f$ upwind model, while the model provided by the matrix-fracture fluxes (23)(24) is termed in the following the hybrid-dimensional $m f$ linear $m$ upwind model.

Remark 3.1 (Non homogeneous matrix and fracture network) We remark that the discrete fluxes defined by (15) and (16) make use of elimination of interface potential from the linear flux continuity equations (13). This is indeed possible because we have neglected the jump of the saturation across the interface. In order to extend the numerical scheme for the case of cells (and fracture faces) having different capillary pressure-saturation and relative permeability curves one may use the same methodology that has been applied at matrix fracture interfaces. That is instead of (14) and (15), one defines

$$
V_{K \sigma}^{\alpha, n}=T_{K \sigma}\left(w_{K}^{\alpha, n}-w_{\sigma}^{\alpha, n}\right),
$$

and

$$
F_{K \sigma}^{\alpha, n}=k_{m}^{\alpha}\left(s_{K}^{\alpha, n}, \mathbf{x}_{K}\right)\left(V_{K \sigma}^{\alpha, n}\right)^{+}+k_{m}^{\alpha}\left(s_{K \sigma}^{\alpha, n}, \mathbf{x}_{K}\right)\left(V_{K \sigma}^{\alpha, n}\right)^{-},
$$

with

$$
s_{K \sigma}^{\alpha, n}=S_{m}^{\alpha}\left(w_{\sigma}^{n w, n}-w_{\sigma}^{w, n}-\left(\rho^{n w}-\rho^{w}\right) g z_{\sigma}, \mathbf{x}_{K}\right) .
$$

The flux is computed by solving (12) for $\alpha=w, n w$ with respect to $w_{\sigma}^{n w, n}$ and $w_{\sigma}^{w, n}$, which results in a couple of nonlinear equations. The same approach can be applied at fracture-fracture interfaces.

Remark 3.2 (Continuous representation of the linear transmission conditions) The equations (17)-(19) reproduce at the discrete level the coupling condition (8). Let us recover, in a formal way, the continuous coupling condition, which would correspond to the discrete mf flux defined by (21) or (23) instead of (20). Let $\sigma \in \mathcal{F}_{\Gamma_{i}}, i \in I$ and let $K \in \mathcal{M}_{\sigma}$ be such $K$ lies on the "+" side of $\Gamma_{i}$ (the argumentation is similar for the "-" side). Using (21) or (23) we write the discrete flux as

$$
F_{K \sigma}^{\alpha, n}=k_{K \sigma}^{\alpha, n} \frac{T_{K \sigma} T_{\sigma}}{T_{K \sigma}+T_{\sigma}}\left(w_{K}^{\alpha, n}-w_{\sigma}^{\alpha, n}\right)
$$

where $k_{K \sigma}^{\alpha, n}$ is an appropriate upstream mobility defined for the linear $\mathrm{f}$ upwind model as

$$
k_{K \sigma}^{\alpha, n}= \begin{cases}k_{m}^{\alpha}\left(s_{K}^{\alpha, n}\right) & \text { if } V_{K \sigma}^{\alpha, n} \geq 0 \\ k_{f}^{\alpha}\left(s_{\sigma}^{\alpha, n}\right) & \text { else }\end{cases}
$$


and for the linear $\mathrm{m}$ upwind model by

$$
k_{K \sigma}^{\alpha, n}= \begin{cases}k_{m}^{\alpha}\left(s_{K}^{\alpha, n}\right) & \text { if } V_{K \sigma}^{\alpha, n} \geq 0 \\ k_{m}^{\alpha}\left(s_{K \sigma}^{\alpha, n}\right) & \text { else }\end{cases}
$$

with $s_{K \sigma}^{\alpha, n}$ given by (24). The transmissibility may be written as

$$
\frac{T_{K \sigma} T_{\sigma}}{T_{K \sigma}+T_{\sigma}}=\frac{|\sigma| \lambda_{f, n}\left(\mathbf{x}_{\sigma}\right)}{d_{f}\left(\mathbf{x}_{\sigma}\right) / 2+\frac{\lambda_{f, n}}{\Lambda_{m}\left(\mathbf{x}_{K}\right)}\left(\mathbf{x}_{\sigma}\right)\left|\mathbf{x}_{K}-\mathbf{x}_{\sigma}\right|}
$$

so that $\frac{T_{K \sigma} T_{\sigma}}{T_{K \sigma}+T_{\sigma}}$ tends to $\frac{|\sigma| \lambda_{f, n}\left(\mathbf{x}_{\sigma}\right)}{d_{f}\left(\mathbf{x}_{\sigma}\right) / 2}$ when $\mathbf{x}_{K} \rightarrow \mathbf{x}_{\sigma}$. When the mesh size and the time step are small one would expect the discrete flux $F_{K \sigma}^{\alpha, n}$ to be an approximation of the quantity

$$
\frac{1}{\Delta t_{n}} \int_{t_{n-1}}^{t_{n}} \int_{\sigma} k_{m f}^{\alpha,+} V_{f, i, n}^{\alpha,+} d \sigma(\mathbf{x}) d t
$$

where

$$
k_{m f}^{\alpha,+}= \begin{cases}k_{m}^{\alpha}\left(S_{m}^{\alpha}\left(\gamma_{i}^{+} p_{m}^{n w}-\gamma_{i}^{+} p_{m}^{w}\right)\right) & \text { if } V_{f, i, n}^{\alpha,+} \geq 0 \\ \left.k_{f}^{\alpha}\left(S_{f}^{\alpha}\left(p_{f}^{n w}-p_{f}^{w}\right)\right)\right) & \text { else }\end{cases}
$$

for the linear $\mathrm{f}$ upwind model, and

$$
k_{m f}^{\alpha,+}= \begin{cases}k_{m}^{\alpha}\left(S_{m}^{\alpha}\left(\gamma_{i}^{+} p_{m}^{n w}-\gamma_{i}^{+} p_{m}^{w}\right)\right) & \text { if } V_{f, i, n}^{\alpha,+} \geq 0 \\ \left.k_{m}^{\alpha}\left(S_{m}^{\alpha}\left(p_{f}^{n w}-p_{f}^{w}\right)\right)\right) & \text { else }\end{cases}
$$

for the linear $\mathrm{m}$ upwind model. We then deduce that, for $i \in I$, the continuous coupling condition corresponding to $\mathrm{f}$ and $\mathrm{m}$ linear upwind models would be respectively

$$
\left.\mathbf{q}_{m}^{\alpha, \pm} \cdot \mathbf{n}_{i}^{ \pm}=k_{m}^{\alpha}\left(S_{m}^{\alpha}\left(\gamma_{i}^{ \pm} p_{m}^{n w}-\gamma_{i}^{ \pm} p_{m}^{w}\right)\right)\left(V_{f, i, n}^{\alpha, \pm}\right)^{+}+k_{f}^{\alpha}\left(S_{f}^{\alpha}\left(p_{f}^{n w}-p_{f}^{w}\right)\right)\right)\left(V_{f, i, n}^{\alpha, \pm}\right)^{-}
$$

and

$$
\left.\mathbf{q}_{m}^{\alpha, \pm} \cdot \mathbf{n}_{i}^{ \pm}=k_{m}^{\alpha}\left(S_{m}^{\alpha}\left(\gamma_{i}^{ \pm} p_{m}^{n w}-\gamma_{i}^{ \pm} p_{m}^{w}\right)\right)\left(V_{f, i, n}^{\alpha, \pm}\right)^{+}+k_{m}^{\alpha}\left(S_{m}^{\alpha}\left(p_{f}^{n w}-p_{f}^{w}\right)\right)\right)\left(V_{f, i, n}^{\alpha, \pm}\right)^{-}
$$

\section{Nonlinear interface problem}

In this section, we present the algorithm for solving the local nonlinear interface problem (19) with respect to the unknowns $\left(w_{K \sigma}^{\alpha, n}\right)_{\alpha=w, n w}$.

Let $T_{m}, T_{f}>0$ and let us define for all $\pi \in \mathbb{R}$

$$
M_{m}^{\alpha}(\pi)=T_{m} k_{m}^{\alpha}\left(S_{m}^{\alpha}(\pi)\right) \quad \text { and } \quad M_{f}^{\alpha}(\pi)=T_{f} k_{f}^{\alpha}\left(S_{f}^{\alpha}(\pi)\right) .
$$

In an abstract form, the local interface problem may be reformulated as follows:

Let $\left(w_{j}^{\alpha}\right)_{j=m, f}^{\alpha=w, n w}, z_{m}, z_{f}$ and $z_{m f}$ be given and let us denote by $\pi_{j}$, for $j=m, f$, the capillary pressure

$$
\pi_{j}=w_{j}^{n w}-w_{j}^{w}-\left(\rho^{n w}-\rho^{w}\right) g z_{j} .
$$

We look for a triplet $\left(w^{n w}, w^{w}, \pi\right)$ satisfying

$$
M_{m, m f}^{\alpha}\left(w_{m}^{\alpha}-w^{\alpha}\right)+M_{f, m f}^{\alpha}\left(w_{f}^{\alpha}-w^{\alpha}\right)=0 \quad \text { for } \alpha=w, n w
$$

and

$$
\pi=w^{n w}-w^{w}-\left(\rho^{n w}-\rho^{w}\right) g z_{m f},
$$


where, for $j=m, f$, we set

$$
M_{j, m f}^{\alpha}=\left\{\begin{array}{lll}
M_{j}^{\alpha}\left(\pi_{j}\right) & \text { if } \quad w_{j}^{\alpha}-w^{\alpha}>0 \\
M_{j}^{\alpha}(\pi) & \text { if } \quad w_{j}^{\alpha}-w^{\alpha} \leq 0 .
\end{array}\right.
$$

Let us remark that capillary pressures $\pi$ smaller then the minimal entry pressure $\pi_{\min }=\min \left(\pi_{m, e}, \pi_{f, e}\right)$ are not considered since for $\pi \leq \pi_{m i n}$ it holds that $\partial_{\pi} M_{m}^{\alpha}(\pi)=\partial_{\pi} M_{f}^{\alpha}(\pi)=0, \alpha=w, n w$.

We present below an algorithm for finding a solution to (27)-(28). Let us briefly explain the idea behind it. It is assumed first that, for both phases, each of the equation (27) can be uniquely solved with respect to the corresponding $w^{\alpha}$. This means that there exists a pair of functions $W^{\alpha}(\pi)$, $\alpha=w, n w$, such that, for a given $\pi \geq \pi_{\min }$, the couple $\left(W^{\alpha}(\pi), \pi\right)$ constitute the unique solution of (27). Moreover, for practical purposes one has to be able to compute $W^{\alpha}(\pi)$ (and probably even $\left.\left(W^{\alpha}\right)^{\prime}(\pi)\right)$. The system (27)-(28) is then reduced to the equation

$$
W^{n w}(\pi)=\widehat{W}^{n w}(\pi)
$$

where $\widehat{W}^{n w}(\pi)$ denotes the non-wetting phase potential based on $W^{w}(\pi)$ and the equation (28), that is

$$
\widehat{W}^{n w}(\pi)=W^{w}(\pi)+\pi-\left(\rho^{w}-\rho^{n w}\right) g z_{m f} .
$$

The equation (30) has to be solved numerically using some iterative method.

As it is going to be shown below, the function $W^{w}$ is well defined and is continuous. In contrast, the values of $W^{n w}(\pi)$ are not in general uniquely defined. It turns out that in certain situations the set of $w^{n w}$ satisfying (27) with $\pi=\pi_{\min }$ is a half-line. This is due to the fact that the non-wetting phase may vanish. The structure of the non-wetting phase balance equation (27) is dictated by the presence of the concerned phase on the adjacent matrix and fracture sides of the interface. We will distinguish three following cases:

- Single phase flow characterized by an absence of the non-wetting phase on both sides of the interface.

- Degenerate two-phase flow taking place when the non-wetting phase is missing exclusively on the upwind side.

- Non-degenerate two-phase flow taking place when the non-wetting phase is present on the upwind side.

Now let us give a detailed description of the algorithm. Using the notations

$$
(\operatorname{up}(\alpha), \operatorname{do}(\alpha))=\left\{\begin{array}{lll}
(m, f) & \text { if } \quad w_{m}^{\alpha} \geq w_{f}^{\alpha}, \\
(f, m) & \text { if } \quad w_{f}^{\alpha}>w_{m}^{\alpha} .
\end{array}\right.
$$

we may rewrite $(27)$ as

$$
M_{\mathrm{up}(\alpha), m f}^{\alpha}\left(w_{\mathrm{up}(\alpha)}^{\alpha}-w^{\alpha}\right)+M_{\mathrm{do}(\alpha), m f}^{\alpha}\left(w_{\operatorname{do}(\alpha)}^{\alpha}-w^{\alpha}\right)=0 \quad \text { for } \alpha=w, n w .
$$

Since the wetting phase mobility does not vanish, the solution of the equation (31) for $\alpha=w$ at given $\pi$ is defined by

$$
w^{w}=W^{w}(\pi)
$$

with

$$
W^{w}(\pi)=w_{\mathrm{do}(w)}^{w}+M_{\mathrm{up}(w)}^{w}\left(\pi_{\mathrm{up}(w)}\right) \frac{w_{\mathrm{up}(w)}^{w}-w_{\mathrm{do}(w)}^{w}}{M_{\mathrm{up}(w)}^{w}\left(\pi_{\mathrm{up}(w)}\right)+M_{\mathrm{do}(w)}^{w}(\pi)} .
$$

Let us remark that the function $W^{w}$ is non-decreasing and continuous on $\mathbb{R}$, so that

$$
\widehat{W}^{n w}(\pi)=W^{w}(\pi)+\pi-\left(\rho^{w}-\rho^{n w}\right) g z_{m f}
$$


is a strictly increasing and continuous function on $\mathbb{R}$. Now, we look for $w^{n w}$ and $\pi \in\left[\pi_{m i n},+\infty\right)$ satisfying

$$
w^{n w}=\widehat{W}^{n w}(\pi)
$$

and the equation (31) for the non-wetting phase $\alpha=n w$. Since the non-wetting phase mobility may vanish, we consider three following cases.

Single-phase case: If the non-wetting phase is missing at both sides of the interface, i.e $M_{m}^{n w}\left(\pi_{m}\right)=$ $M_{f}^{n w}\left(\pi_{f}\right)=0$, we set

$$
\pi=\pi_{m i n}, w^{w}=W^{w}\left(\pi_{m i n}\right) \text { and } w^{n w}=\widehat{W}^{n w}\left(\pi_{m i n}\right) .
$$

Degenerate two-phase case: If the non-wetting phase is missing only on the upwind side, that is $M_{\mathrm{up}(n w)}^{n w}\left(\pi_{\mathrm{up}(n w)}\right)=0$ and $M_{\mathrm{do}(n w)}^{n w}\left(\pi_{\mathrm{do}(n w)}\right)>0$, the equation (31) for $\alpha=n w$ is equivalent to either

$$
\pi=\pi_{\min } \text { and } w^{n w}>w_{\mathrm{up}(n w)}^{n w},
$$

or

$$
M_{\mathrm{do}(n w), m f}^{n w}\left(w_{\mathrm{do}(n w)}^{n w}-w^{n w}\right)=0 \text { and } w^{n w} \leq w_{\mathrm{up}(n w)}^{n w} .
$$

Moreover since $M_{\mathrm{do}(n w)}^{n w}\left(\pi_{\mathrm{do}(n w)}\right)>0$, the equation (34) implies that $w^{n w} \geq w_{\mathrm{do}(n w)}^{n w}$ and $M_{\mathrm{do}(n w), m f}^{n w}=$ $M_{\mathrm{do}(n w)}^{n w}(\pi)$. Hence, the equation (34) is equivalent to

$$
M_{\mathrm{do}(n w)}^{n w}(\pi)\left(w_{\mathrm{do}(n w)}^{n w}-w^{n w}\right)=0 \text { and } w^{n w} \leq w_{\mathrm{up}(n w)}^{n w} .
$$

Let us consider the following complementary problem

$$
\left\{\begin{array}{l}
\left(\pi-\pi_{\min }\right)\left(w^{n w}-w_{\mathrm{do}(n w)}^{n w}\right)=0 \\
\pi \geq \pi_{\min }, \quad w^{n w} \geq w_{\mathrm{do}(n w)}^{n w}
\end{array}\right.
$$

One can see that any solution of (36) satisfies (33) or (35). Let us notice that the system formed by (32) and (36) has a unique solution. In order to find it we proceed as follows (see also Figure 2):

- If $\widehat{W}^{n w}\left(\pi_{\min }\right)>w_{\mathrm{do}(n w)}^{n w}$ we set $\pi=\pi_{\min }, w^{w}=W^{w}\left(\pi_{\min }\right)$ and $w^{n w}=\widehat{W}^{n w}\left(\pi_{\min }\right) ;$

- If not, $\pi$ is the unique solution of $\widehat{W}^{n w}(\pi)=w_{\mathrm{do}(n w)}^{n w}$, and we set $w^{w}=W^{w}(\pi)$ and $w^{n w}=$ $w_{\operatorname{do}(n w)}^{n w}$.

Non-degenerate two-phase case: Finally, if $M_{\mathrm{up}(n w)}^{n w}\left(\pi_{\mathrm{up}}(n w)\right)>0$ we define

$$
W^{n w}(\pi)=w_{\mathrm{do}(n w)}^{n w}+M_{\mathrm{up}(n w)}^{n w}\left(\pi_{\mathrm{up}(n w)}\right) \frac{w_{\mathrm{up}(n w)}^{n w}-w_{\mathrm{do}(n w)}^{n w}}{M_{\mathrm{up}(n w)}^{n w}\left(\pi_{\mathrm{up}(n w)}\right)+M_{\mathrm{do}(n w)}^{n w}(\pi)},
$$

where $W^{n w}(\pi)$ is a non-increasing function on $\left[\pi_{\min },+\infty\right)$ such that $W^{n w}\left(\pi_{\min }\right)=w_{\mathrm{up}(n w)}^{n w}$. One can verify that the pair $\left(w^{n w}, \pi\right)$ is the solution of (31) for $\alpha=n w$ (excluding $\pi<\pi_{\min }$ ) if and only if it satisfies

$$
\left\{\begin{array}{l}
\left(\pi-\pi_{\min }\right)\left(w^{n w}-W^{n w}(\pi)\right)=0, \\
\pi \geq \pi_{\min }, \quad w^{n w} \geq W^{n w}(\pi) .
\end{array}\right.
$$

Again the system formed by (32) and (37) has a unique solution and this solution is found by the following procedure (see Figure 2):

$$
\text { - If } \widehat{W}^{n w}\left(\pi_{m i n}\right)>w_{\mathrm{up}(n w)}^{n w} \text { we set } \pi=\pi_{m i n}, w^{w}=W^{w}\left(\pi_{m i n}\right) \text { and } w^{n w}=\widehat{W}^{n w}\left(\pi_{m i n}\right) ;
$$


- If not, $\pi$ is the unique solution of $W^{n w}(\pi)=\widehat{W}^{n w}(\pi)$, and we set $w^{w}=W^{w}(\pi)$ and $w^{n w}=$ $W^{n w}(\pi)$.
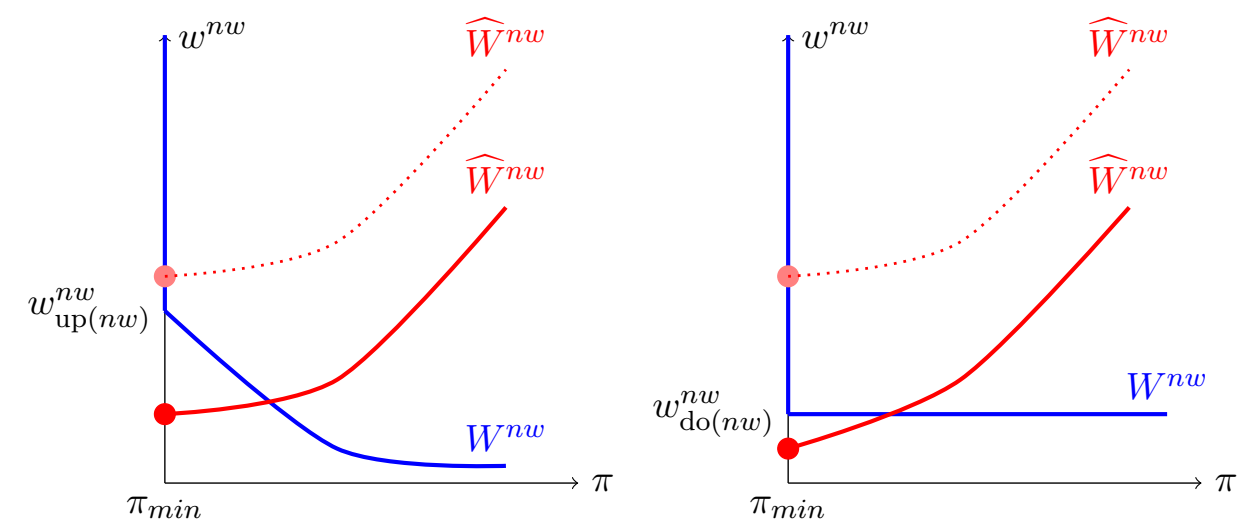

Figure 2: The values of $W^{n w}$ and $\widehat{W}^{n w}$ versus capillary pressure $\pi$ in non-degenerate (left) and degenerate (right) two phase cases.

\section{$5 \quad 2 \mathrm{D}$ numerical experiments}

The objective of this section is to compare on 2D test cases, both in terms of accuracy and CPU time, the numerical solutions obtained with the hybrid-dimensional $m f$ nonlinear and $m f$ linear $f$ upwind and $m$ upwind models. The reference solution is defined by the equi-dimensional numerical solution obtained with 4 cells in the width of the fractures. The test cases presented in the following subsections consider various physical configurations including gravity, viscous or capillary dominant test cases with homogeneous or heterogeneous capillary pressures, and with highly or low permeable fractures.

The discretization is based on the Two-Point Flux Approximation defined in Section 3 using uniform Cartesian meshes. The fluxes at the matrix-fracture interfaces for the equi-dimensional model are discretized using (25)-(26) rather than (14)-(15) as mentioned in Remark 3.1 in order to account for the saturation jump accurately. This discretization is combined with the nonlinear interface solver introduced in Section 4 both for the hybrid-dimensional $m f$ nonlinear model at matrice fracture interfaces and for the equi-dimensional model at interfaces between the matrix and fracture rocktypes.

The following notations and numerical parameters are used in this section for all the simulations. Let $t_{f}$ denote the final time of the simulation, and let be given the initial time step $\Delta t^{1}$, a maximum time step $\Delta t^{\max }$, and an objective variation of the saturation between two successive time steps $\left.\left.\delta s^{o b j} \in\right] 0,1\right]$. The time stepping is adaptive and defined by

$$
\Delta t^{n+1}=\max \left(\beta \Delta t^{n}, \Delta t^{\max }\right)
$$

with $\beta=\min \left(1.2, \frac{\delta s^{o b j}}{\delta s^{n}}\right)$ where $\delta s^{n}$ is the maximum variation of the saturation at time step $n$. At each time step, the set of nonlinear equations (9)-(10)-(11) is solved by a Newton-Raphson algorithm using the non-wetting phase pressure and non-wetting phase saturation as primary unknowns. This choice is known to provide a better nonlinear convergence than the one obtained with both phase pressures as primary unknowns. It can also allow vanishing capillary pressure curves. At each NewtonRaphson iteration, the Jacobian is computed analytically and the linear system is solved using a GMRes iterative solver combined with the Constrained Pressure Residual Algebraic Multigrid (CPRAMG) right preconditioner $[22,26]$. The time step is chopped by a factor 2 in case the Newton-Raphson algorithm has not converged in 50 iterations with the Newton-Raphson stopping criteria defined by 
the relative norm of the residual lower than $10^{-6}$ or a maximum normalized variation of the primary unknowns lower than $10^{-5}$.

In the tables below, the following notations are used:

- Nb Cells is the number of cells of the mesh,

- $\mathbf{N b}$ dof is the number of degrees of freedom (d.o.f.) (with two physical primary unknowns per d.o.f.) including in addition to the cells, the faces $\sigma \in \mathcal{F}_{\Gamma}$ and the edges $e \in \mathcal{E}_{\star}$ for the hybrid-dimensional models,

- $\mathbf{N}_{\Delta t}$ is the number of successful time steps and $\mathbf{N}_{C h o p}$ is the number of time step chops,

- $\mathbf{N}_{\text {Newton }}$ is the average number of Newton-Raphson iterations per successful time step,

- $\mathbf{N}_{\text {GM Res }}$ is the average number of GMRes iterations per Newton-Raphson step using the stopping criteria $10^{-7}$ on the relative residual.

\subsection{Gravity dominant flow with homogeneous capillary pressures}

We consider in this test case an oil (o) water (w) two-phase Darcy flow in the DFM model exhibited in Figure 3 and defined by the domain

$$
\Omega=\left\{\left(x=x^{\prime} \cos (\theta)-y^{\prime} \sin (\theta), y=x^{\prime} \sin (\theta)+y^{\prime} \cos (\theta)\right) \mid\left(x^{\prime}, y^{\prime}\right) \in(0,100 \mathrm{~m})^{2}\right\},
$$

and the fracture network $\Gamma=\bigcup_{i=1}^{4} \Gamma_{i}$ with

$$
\begin{aligned}
& \Gamma_{1}=\left\{\left(x=x^{\prime} \cos (\theta)-y^{\prime} \sin (\theta), y=x^{\prime} \sin (\theta)+y^{\prime} \cos (\theta)\right) \mid x^{\prime} \in(0,25), y^{\prime}=25\right\}, \\
& \Gamma_{2}=\left\{\left(x=x^{\prime} \cos (\theta)-y^{\prime} \sin (\theta), y=x^{\prime} \sin (\theta)+y^{\prime} \cos (\theta)\right) \mid x^{\prime} \in(25,75), y^{\prime}=25\right\}, \\
& \Gamma_{3}=\left\{\left(x=x^{\prime} \cos (\theta)-y^{\prime} \sin (\theta), y=x^{\prime} \sin (\theta)+y^{\prime} \cos (\theta)\right) \mid x^{\prime} \in(25,100), y^{\prime}=75\right\}, \\
& \Gamma_{4}=\left\{\left(x=x^{\prime} \cos (\theta)-y^{\prime} \sin (\theta), y=x^{\prime} \sin (\theta)+y^{\prime} \cos (\theta)\right) \mid y^{\prime} \in(0,25), x^{\prime}=25\right\},
\end{aligned}
$$

setting $\theta=\frac{\pi}{12}$.

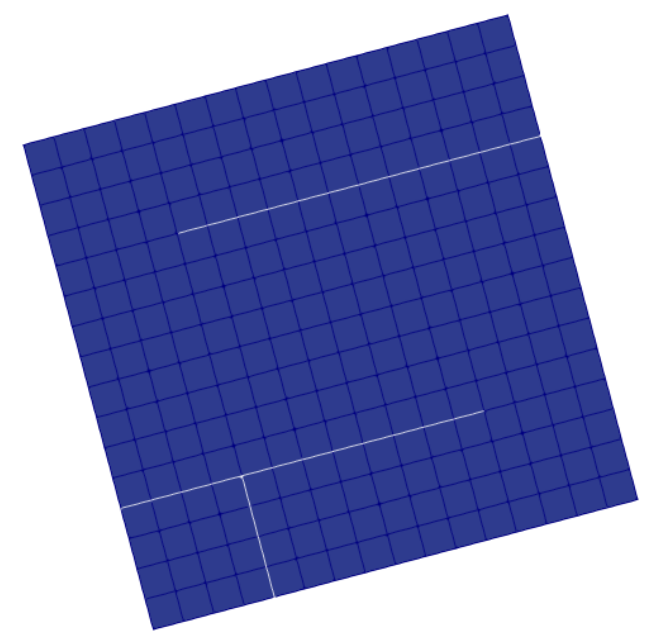

Figure 3: Discrete Fracture Model with the four fractures $\Gamma_{i}, i=1, \cdots, 4$, and its uniform Cartesian mesh of size $16 \times 16$.

The matrix and the fracture network have the same relative permeabilities and capillary pressures given by $k_{r, f}^{o}\left(s^{o}\right)=k_{r, m}^{o}\left(s^{o}\right)=\left(s^{o}\right)^{2}, k_{r, f}^{w}\left(s^{w}\right)=k_{r, m}^{w}\left(s^{w}\right)=\left(s^{w}\right)^{2}$ and by $P_{c, m}\left(s^{o}\right)=P_{c, f}\left(s^{o}\right)=$ $-b \log \left(1-s^{o}\right)$ with $b=10 \mathrm{~Pa}$. The matrix is homogeneous and charaterized by the isotropic permeability $\Lambda_{m}=0.1$ Darcy, and the porosity $\phi_{m}=0.2$. The fracture properties are set to $d_{f}=1$ 
$\mathrm{cm}, \Lambda_{f}=\lambda_{f, n}=10^{3} \Lambda_{m}, \phi_{f}=0.35$. The fluid properties are defined by their dynamic viscosities $\mu^{o}=5.10^{-3}, \mu^{w}=10^{-3}$ Pa.s and their mass densities $\rho^{w}=1000$ and $\rho^{o}=800 \mathrm{Kg} . \mathrm{m}^{-3}$.

The matrix and fracture domains are initially saturated by the water phase. The top boundary conditions at $y^{\prime}=100$ are defined by the water saturation $s^{w}=1$ and the water pressure

$$
p^{w}(x, y)=(100(\cos (\theta)+\sin (\theta))-y) g \rho^{w} \mathrm{~Pa} .
$$

The bottom boundary conditions at $y^{\prime}=0$ are impervious in the matrix and defined in the bottom fracture by the water pressure

$$
p^{w}=(100(\cos (\theta)+\sin (\theta))-25 \sin (\theta)) g \rho^{w} \mathrm{~Pa},
$$

and the oil saturation $s^{o}=0.9$. The lateral boundaries are assumed impervious.

The simulation time is set to $t_{f}=35$ years. The time stepping is defined by the parameters $\Delta t^{\max }=30$ days, $\Delta t^{1}=0.01$ day, and $\delta s^{o b j}=0.5$.

Figure 4 compares the solutions obtained with the hybrid-dimensional $m f$ nonlinear and $m f$ linear models to a reference solution obtained using the equi-dimensional model meshed with 4 cells in the fracture width and a Cartesian grid of size $260 \times 264$ uniform in the matrix domain. The solutions of the hybrid-dimensional $m f$ nonlinear and $m f$ linear models are obtained using a uniform Cartesian grid of size $256 \times 256$. Note that the hybrid-dimensional $m f$ linear $m$ and $f$ upwind models provide the same solution since the relative permeabilities and the capillary pressures are the same in the matrix and fracture domains for this test case.

Figure 4 shows that the hybrid-dimensional $m f$ nonlinear model reproduces accurately the reference solution provided by the equi-dimensional model while the hybrid-dimensional $\mathrm{mf}$ linear model exhibits large differences with the reference solution. This discrepancy does not reduce when the mesh is refined and is due to the fact that the hybrid-dimensional $m f$ linear model cannot account for gravity segregation in the fracture width since it uses a single saturation unknown in the fracture width. As a consequence, the top mobility of oil rising out of the fractures is averaged and reduced for the $m f$ linear model. On the other hand, the hybrid-dimensional $m f$ nonlinear model accounts for gravity segregation thanks to its additional saturation unknowns at the $m f$ interfaces and provides a good approximation of the top $m f$ interface oil mobility. It can be checked that this discrepancy is considerably reduced for a larger capillary pressure in the fractures (say for $b=1000 \mathrm{~Pa}$ ) which smoothes out the gravity segregation in the fracture width as exhibited in Figure 5. Table 1 exhibits a gain of a factor 4 in CPU time between the equi-dimensional and the hybrid-dimensional $m f$ nonlinear model. This is mainly due to a higher number of GMRes iterations for the equi-dimensional model which can be explained by the smaller volumes in the fracture width and at the fracture intersection. The hybrid-dimensional $m f$ nonlinear model is only 50 percent more expensive than the hybrid-dimensional $m f$ linear model due to a higher number of nonlinear iterations. 


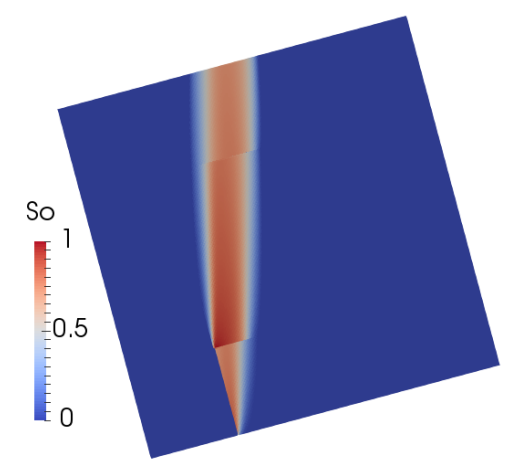

(a) Equi-dimensional

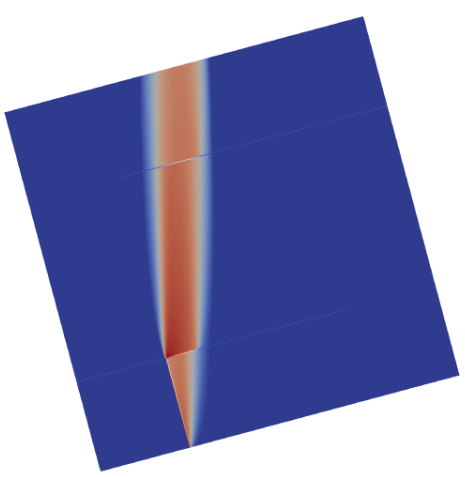

(b) $m f$-nonlinear

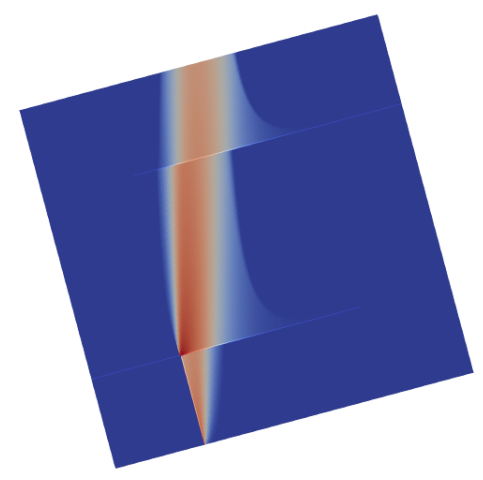

(c) linear $m$ - or $f-$ upwind

Figure 4: Oil saturation at final time computed by the equi-dimensional and hybrid-dimensional models for the gravity dominant test case with homogeneous capillary pressures. The mesh is Cartesian of size $260 \times 264$ for the equi-dimensional model and of size $256 \times 256$ for the hybrid-dimensional models.
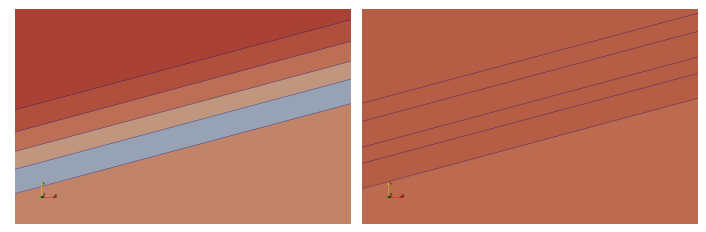

Figure 5: Zoom on the oil saturation in the $\Gamma_{2}$ fracture width for the equi-dimensional model with $b=10 \mathrm{~Pa}$ (left) and $b=1000 \mathrm{~Pa}$ (right).

\begin{tabular}{|c|c|c|c|c|c|c|c|}
\hline Model & Nb Cells & Nb dof & $\mathbf{N}_{\Delta t}$ & $\mathbf{N}_{\text {Newton }}$ & $\mathbf{N}_{\text {GMRes }}$ & $\mathbf{N}_{\text {Chop }}$ & $\mathbf{C P U [ s ]}$ \\
\hline equi-dimensional & 68640 & 68640 & 471 & 4.5 & 55 & 4 & 2574 \\
\hline$m f$ nonlinear & 65536 & 65985 & 463 & 4.0 & 11 & 2 & 612 \\
\hline$m f$ linear & 65536 & 65985 & 459 & 3.0 & 10.3 & 0 & 436 \\
\hline
\end{tabular}

Table 1: Numerical behavior of the equi-dimensional and hybrid-dimensional models for the gravity dominant test case with homogeneous capillary pressures. The mesh is Cartesian of size $260 \times 264$ for the equi-dimensional model and of size $256 \times 256$ for the hybrid-dimensional models.

\subsection{Gravity dominant flow with heterogeneous capillary pressures}

We consider the same test case as in the previous subsection with different relative permeabilities and capillary pressures in the matrix and in the fracture network defined by

$$
k_{r, f}^{o}\left(s^{o}\right)=s^{o}, \quad k_{r, m}^{o}\left(s^{o}\right)=\left(s^{o}\right)^{2}, \quad k_{r, f}^{w}\left(s^{w}\right)=s^{w}, \quad k_{r, m}^{w}\left(s^{w}\right)=\left(s^{w}\right)^{2},
$$

and

$$
P_{c, m}\left(s^{o}\right)=10^{3}\left(1-\log \left(1-s^{o}\right)\right) \mathrm{Pa}, \quad P_{c, f}\left(s^{o}\right)=-10 \log \left(1-s^{o}\right) \mathrm{Pa} .
$$

The simulation time is reduced to $t_{f}=20$ years for this test case, and the time stepping is defined by the parameters $\Delta t^{\max }=30$ days, $\Delta t^{1}=0.01$ day, and $\delta s^{o b j}=0.5$. Other physical and numerical parameters are unchanged compared with the previous test case.

Figure 6 compares the solutions obtained using the hybrid-dimensional $m f$ nonlinear and $m f$ linear $f$ and $m$ upwind models to the reference solution obtained using the equi-dimensional model with 4 
cells in the fracture width. Two different Cartesian mesh sizes are used, $64 \times 64(68 \times 72$ for the equi-dimensional model $)$ and $256 \times 256(260 \times 264$ for the equi-dimensional model $)$. The hybriddimensional $m f$ linear $f$ upwind model overestimates the oil flux rising out of the fractures for the coarsest mesh. This is due to the absence of an $m f$ interface capillary pressure unknown for this model and to a cell size above the fracture not small enough compared with the entry pressure divided by the gravity constant $g$ and by $\rho^{w}-\rho^{o}$. To be more specific, this model overestimates the buoyancy force at the top matrix fracture interface by the quantity $g\left(\rho^{w}-\rho^{o}\right) \frac{\Delta z}{2}$ where $\Delta z$ is the cell height above the fracture. If this quantity is not small compared with the matrix entry pressure, this model will overestimate the oil flux rising out of the fractures. This is clearly observed on the coarsest mesh for which $g\left(\rho^{w}-\rho^{o}\right) \frac{\Delta z}{2} \simeq 780 \mathrm{~Pa}$ which is of the same order of magnitude than the matrix entry pressure of $10^{3} \mathrm{~Pa}$. When the mesh is refined by a factor 4 in each direction the discrepancy is much smaller. The $m f$ linear $m$ upwind model is much better for this test case since it better captures the saturation jump at the $m f$ interface. The $m f$ nonlinear model cannot be distinguished from the reference model. Regarding the numerical behavior exhibited in Table 2, on the finest meshes, the $m f$ linear $f$ upwind model is twice cheaper than the $m f$ nonlinear model which is four times cheaper than the equi-dimensional model.

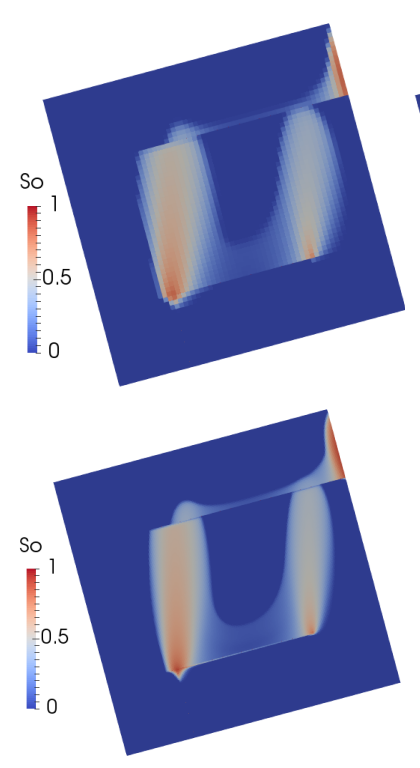

(a) Equi-dim.
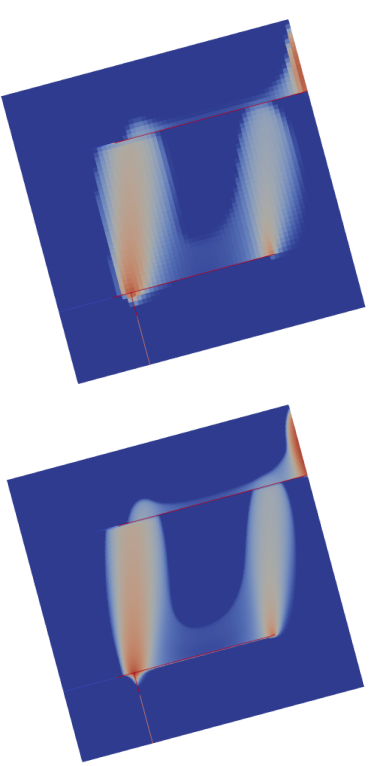

(b) $m f$ non linear
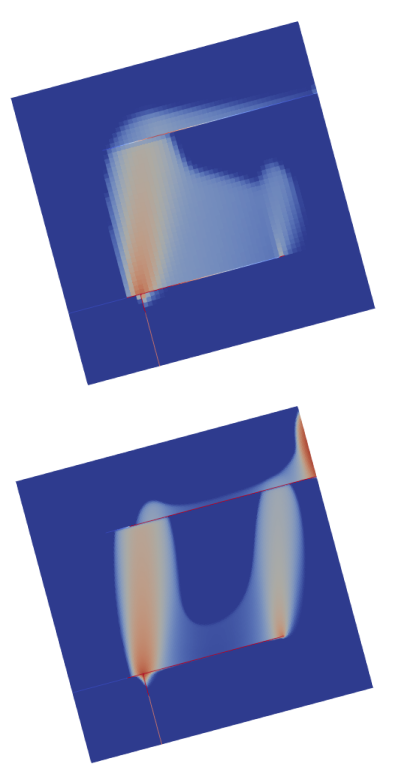

(c) f-upwind
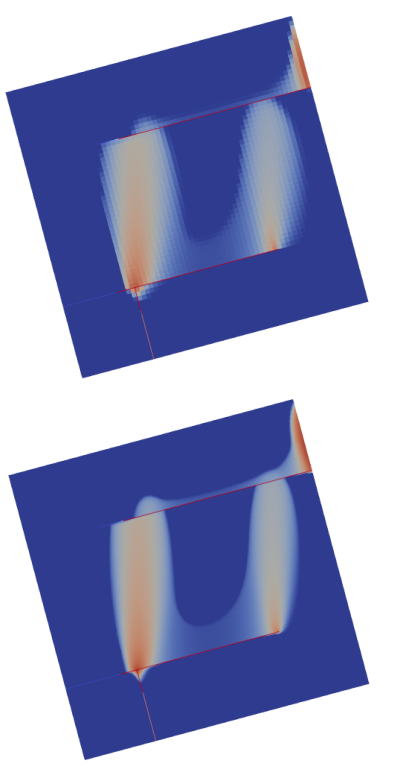

(d) $m$-upwind

Figure 6: From left to right: oil saturation at final time for the equi-dimensional and hybriddimensional models. Top row: Cartesian mesh of size $68 \times 72$ for the equi-dimensional model and of size $64 \times 64$ for the hybrid-dimensional models. Bottom row: Cartesian mesh of size $260 \times 264$ for the equi-dimensional model and of size $256 \times 256$ for the hybrid-dimensional models. 


\begin{tabular}{|c|c|c|c|c|c|c|c|}
\hline Model & Nb Cells & Nb dof & $\mathbf{N}_{\Delta t}$ & $\mathbf{N}_{\text {Newton }}$ & $\mathbf{N}_{\text {GMRes }}$ & $\mathbf{N}_{\text {Chop }}$ & $\mathbf{C P U [ s ]}$ \\
\hline equi-dimensional & 4896 & 4896 & 322 & 9.6 & 17.5 & 6 & 121 \\
\hline$m f$ nonlinear & 4096 & 4209 & 288 & 6.8 & 8.5 & 0 & 47.4 \\
\hline$f$ upwind & 4096 & 4209 & 280 & 3.1 & 8.8 & 0 & 22.7 \\
\hline$m$ upwind & 4096 & 4209 & 289 & 3.5 & 12 & 0 & 28.8 \\
\hline equi-dimensional & 68640 & 68640 & 425 & 13.7 & 42 & 5 & 5040 \\
\hline$m f$ nonlinear & 65536 & 65985 & 337 & 11.7 & 13.3 & 2 & 1296 \\
\hline$f$ upwind & 65536 & 65985 & 326 & 4.9 & 15.6 & 0 & 607 \\
\hline$m$ upwind & 65536 & 65985 & 336 & 4.4 & 28.8 & 0 & 908 \\
\hline
\end{tabular}

Table 2: Numerical behavior of the equi-dimensional and hybrid-dimensional models on both Cartesian meshes for the gravity dominant test case with heterogeneous capillary pressures.

\subsection{Viscous dominant flow with heterogeneous capillary pressures}

We consider the same test case as in the previous subsection with different relative permeabilities and capillary pressures now defined by

$$
k_{r, f}^{o}\left(s^{o}\right)=k_{r, m}^{o}\left(s^{o}\right)=\left(s^{o}\right)^{2}, \quad k_{r, f}^{w}\left(s^{w}\right)=k_{r, m}^{w}\left(s^{w}\right)=\left(s^{w}\right)^{2},
$$

and

$$
P_{c, m}\left(s^{o}\right)=10^{5}\left(1-\log \left(1-s^{o}\right)\right) \mathrm{Pa}, \quad P_{c, f}\left(s^{o}\right)=-100 \log \left(1-s^{o}\right) \mathrm{Pa} .
$$

The water pressure in the bottom fracture at the bottom boundary $y^{\prime}=0$ now includes an overpressure and is set to

$$
p^{w}=2 \cdot 10^{5}+(100(\cos (\theta)+\sin (\theta))-25 \sin (\theta)) g \rho^{w} \text { Pa. }
$$

The simulation time is set to $t_{f}=19$ years for this test case. The time stepping is defined by the parameters $\Delta t^{\max }=30$ days, $\Delta t^{1}=0.01$ day, and $\delta s^{o b j}=1.2 \mathrm{such}$ that $\beta=1.2$. The other physical and numerical parameters are the same as in the previous test case.

We compare in Figure 7 the solutions obtained with the hybrid-dimensional models to the reference solution obtained with the equi-dimensional model with 4 cells in the fracture width. The meshes are Cartesian of size $68 \times 72$ for the equi-dimensional model and of size $64 \times 64$ for the hybrid-dimensional models. For this test case, the $m f$ nonlinear model cannot be distinguished from the reference solution while the $m f$ linear $f$ upwind model slightly overestimates the oil flux rising out of the fractures. This small discrepancy is reduced on a finer mesh. The $m f$ linear $m$ upwind model considerably smoothes out the solution independently of the mesh size as soon as the fractures are filled with oil. As exhibited in Figure 8, this is due to the fact that the capillary pressure inside the filled fractures is too high for the $m$ upwind model which reduces the capillary barrier effect of the matrix. This discrepancy is a modeling error that occurs for filled fractures and increases with the overpressure imposed at the bottom fracture. The numerical behavior of the different models exhibited in Table 3 shows only small differences for this small mesh size. 


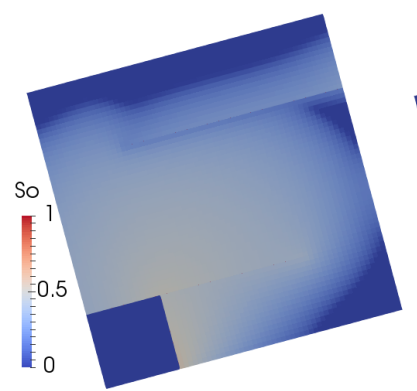

(a) Equi-dim

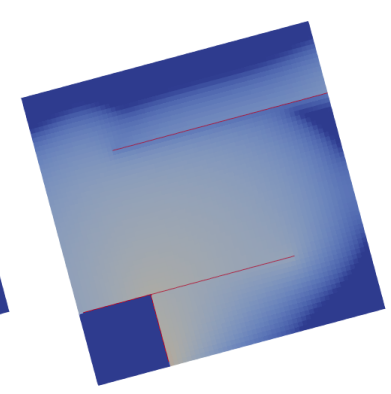

(b) $m f$ nonlinear

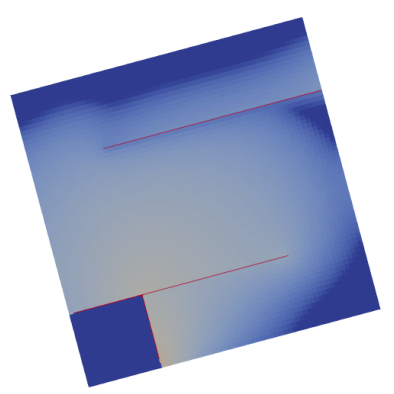

(c) f-upwind

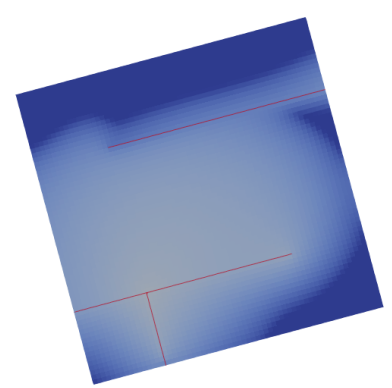

(d) $m$-upwind

Figure 7: From left to right: oil saturation at final time computed by the equi-dimensional and hybrid-dimensional models for the viscous dominant test case with overpressure of $2.10^{5} \mathrm{~Pa}$.

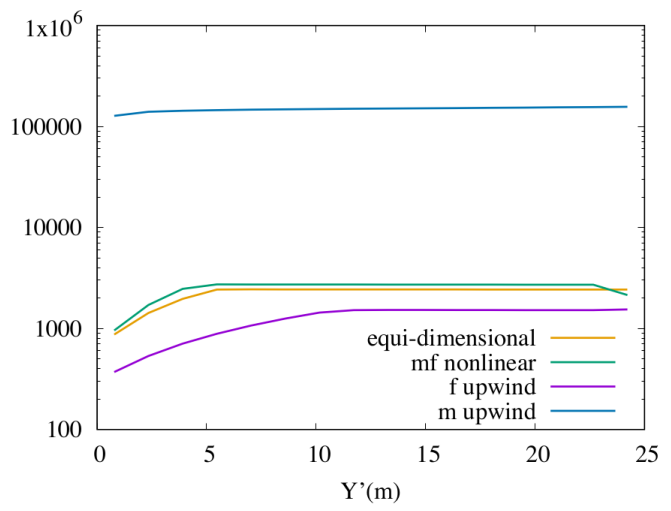

Figure 8: Capillary pressure in $\mathrm{Pa}$ at final time in the bottom fracture $\Gamma_{4}$ as a function of $y^{\prime}$ for the equi-dimensional model (average in the fracture width), the $m f$ nonlinear model, the $m f$ linear $f$ upwind model and the $m f$ linear $m$ upwind model.

\begin{tabular}{|c|c|c|c|c|c|c|c|}
\hline Model & Nb Cells & Nb dof & $\mathbf{N}_{\Delta t}$ & $\mathbf{N}_{\text {Newton }}$ & $\mathbf{N}_{\text {GMRes }}$ & $\mathbf{N}_{\text {Chop }}$ & CPU[s] \\
\hline equi-dimensional & 4896 & 4896 & 269 & 6.0 & 12 & 1 & 56 \\
\hline$m f$ nonlinear & 4096 & 4209 & 269 & 5.6 & 10 & 1 & 36 \\
\hline$f$ upwind & 4096 & 4209 & 267 & 3.8 & 16 & 0 & 31 \\
\hline$m$ upwind & 4096 & 4209 & 267 & 4.0 & 22 & 0 & 40 \\
\hline
\end{tabular}

Table 3: Numerical behavior of the equi-dimensional and the hybrid-dimensional models for the viscous dominant test case with heterogeneous capillary pressures and overpressure of $2.10^{5} \mathrm{~Pa}$. The mesh is Cartesian of size $68 \times 72$ for the equi-dimensional model and of size $64 \times 64$ for the hybrid-dimensional models.

We consider a second viscous dominant test case with one additional fracture

$$
\Gamma_{5}=\left\{\left(x=x^{\prime} \cos (\theta)-y^{\prime} \sin (\theta), y=x^{\prime} \sin (\theta)+y^{\prime} \cos (\theta)\right) \mid y^{\prime} \in(25,50), x^{\prime}=25\right\} .
$$

This test case uses the fracture porosity $\phi_{f}=0.4$, the matrix porosity $\phi_{m}=0.2$, a higher fracture permeability $\Lambda_{f}=\lambda_{f, n}=10^{4}$ Darcy, a lower matrix permeability $\Lambda_{m}=0.01$ Darcy, and a higher overpressure at the bottom boundary of the bottom fracture where the following water pressure is imposed

$$
p^{w}=2 \cdot 10^{6}+(100(\cos (\theta)+\sin (\theta))-25 \sin (\theta)) g \rho^{w} \text { Pa. }
$$


The simulation time is set to $t_{f}=2$ years for this test case. The time stepping is defined by the parameters $\Delta t^{\max }=30$ days, $\Delta t^{1}=0.001$ day, and $\delta s^{o b j}=0.5$. The other physical and numerical parameters are the same as in the previous test case.

We compare in Figures 9 and 10 the solutions obtained with the hybrid-dimensional models to the reference solution obtained with the equi-dimensional model with 4 cells in the fracture width. The meshes are Cartesian of size $260 \times 264$ for the equi-dimensional model and of size $256 \times 256$ for the hybrid-dimensional models. For this test case with a high overpressure compared with the matrix entry pressure, the $m f$ nonlinear model cannot be distinguished from the reference solution while the $m f$ linear $f$ upwind model clearly overestimates the oil flux rising out of the fractures even on this fine mesh. This can be explained by the definition of the matrix-fracture flux for this model which does not take into account the saturation jump at the $m f$ interfaces in contrast to the $m f$ nonlinear and $m f$ linear $m$ upwind models. The $m f$ linear $m$ upwind model provides a very good solution during the fracture filling as can be observed in Figure 10 but again it smoothes out the solution as soon as the fractures are filled with oil. The numerical behavior of the different models exhibited in Table 4 shows that the $m f$ nonlinear model is very competitive compared with the other hybrid-dimensional models with a CPU time almost 3 times lower than the CPU time of the equi-dimensional model.

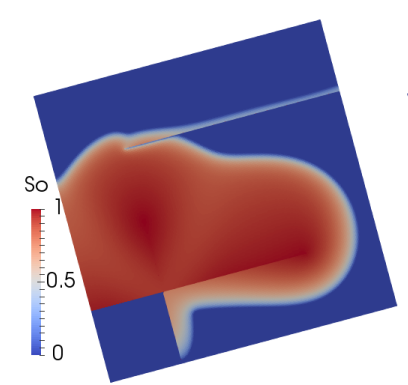

(a) Equi-dim

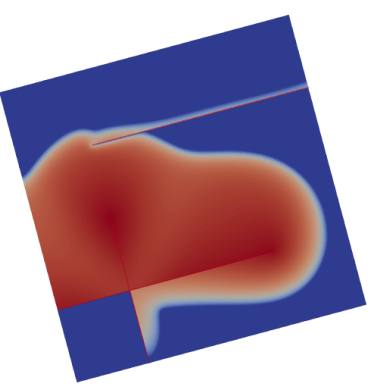

(b) $m f$ nonlinear

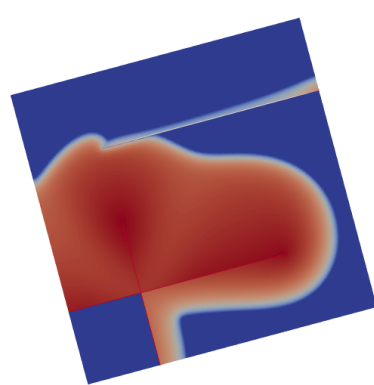

(c) $f$-upwind

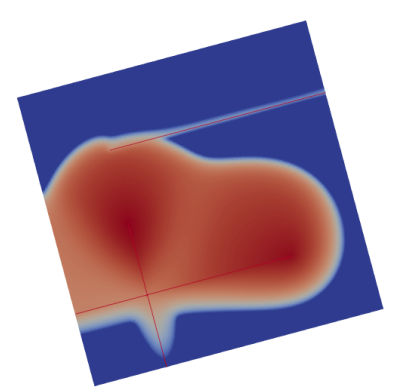

(d) $m$-upwind

Figure 9: From left to right: oil saturation at final time computed by the equi-dimensional and hybrid-dimensional models for the viscous dominant test case with overpressure of $2.10^{6} \mathrm{~Pa}$

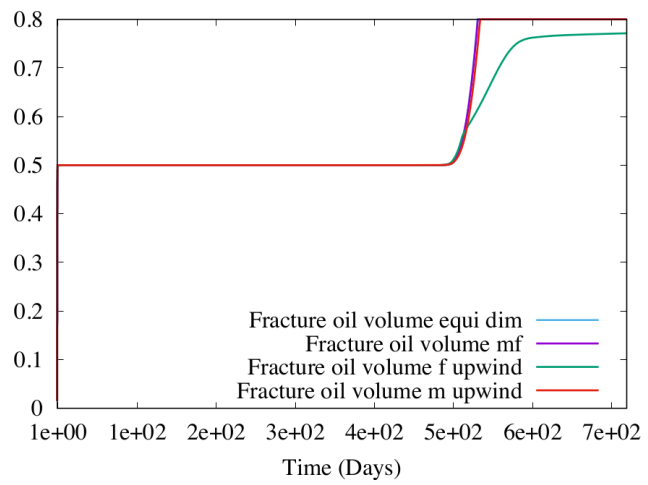

Figure 10: Oil volume in the fracture network as a function of time for the equi-dimensional model, the $m f$ nonlinear and the $m f$ linear $f$ upwind and $m$ upwind models. 


\begin{tabular}{|c|c|c|c|c|c|c|c|}
\hline Model & Nb Cells & Nb dof & $\mathbf{N}_{\Delta t}$ & $\mathbf{N}_{\text {Newton }}$ & $\mathbf{N}_{\text {GMRes }}$ & $\mathbf{N}_{\text {Chop }}$ & $\mathbf{C P U [ s ]}$ \\
\hline equi-dimensional & 68640 & 68640 & 338 & 14.8 & 23 & 16 & 2868 \\
\hline$m f$ nonlinear & 65536 & 66049 & 315 & 12.7 & 8.2 & 11 & 1141 \\
\hline$f$ upwind & 65536 & 66049 & 249 & 14.3 & 8.3 & 27 & 1060 \\
\hline$m$ upwind & 65536 & 66049 & 319 & 13.2 & 12.8 & 9 & 1726 \\
\hline
\end{tabular}

Table 4: Numerical behavior of the equi-dimensional and hybrid-dimensional models for the viscous dominant test case with heterogeneous capillary pressures and overpressure of $2.10^{6} \mathrm{~Pa}$. The mesh is Cartesian of size $260 \times 264$ for the equi-dimensional model and of size $256 \times 256$ for the hybriddimensional models.

\subsection{Three drains and one barrier test case}

In this test case, the matrix properties are set to $\Lambda_{m}=1$ Darcy, $\phi_{m}=0.2$ and $P_{c, m}\left(s^{o}\right)=-5.10^{3}(1-$ $\left.\log \left(1-s^{o}\right)\right)$ Pa. The top fracture $\Gamma_{3}$ is a barrier with properties $\Lambda_{b}=\lambda_{b, n}=10^{-1} \Lambda_{m}, \phi_{b}=0.15$, and $P_{c, b}\left(s^{o}\right)=-10^{4}\left(1-1.5 \log \left(1-s^{o}\right)\right)$ Pa. The bottom fractures $\Gamma_{1}, \Gamma_{2}, \Gamma_{4}$ are drains with properties $\Lambda_{d}=\lambda_{d, n}=10^{3} \Lambda_{m}, \phi_{d}=0.35$, and $P_{c, d}\left(s^{o}\right)=-10^{3} \log \left(1-s^{o}\right)$ Pa. The fracture width is fixed to $d_{f}=1 \mathrm{~cm}$ and the relative permeabilities are set to $k_{r}^{o}\left(s^{o}\right)=\left(s^{o}\right)^{2}$ and $k_{r}^{w}\left(s^{w}\right)=\left(s^{w}\right)^{2}$ for all rocktypes.

The water pressure at the bottom boundary of the bottom fracture is set to

$$
p^{w}=10^{4}+(100(\cos (\theta)+\sin (\theta))-25 \sin (\theta)) g \rho^{w} \mathrm{~Pa} .
$$

The final simulation time is set to $t_{f}=30$ years. The time stepping is defined by the parameters $\Delta t^{\max }=30$ days, $\Delta t^{1}=0.01$ day, and $\delta s^{o b j}=0.5$. Other physical and numerical parameters are the same as in the previous test cases.

We compare in Figure 11 the solutions obtained with the hybrid-dimensional models to a reference solution obtained with the equi-dimensional model with 4 cells in the fracture width. The mesh is Cartesian of size $68 \times 72$ for the equi-dimensional model and of size $64 \times 64$ for the hybrid-dimensional models. As before, the $m f$ nonlinear model cannot be distinguished from the reference model and $m f$ linear $f$ upwind provide a good match with the reference solution for this test case with overpressure. On the other hand, the $m f$ linear $m$ upwind does not capture as expected the capillary barrier effect of the top fracture. The numerical behavior exhibited in Table 5 shows small differences between the hybrid-dimensional models and a factor roughly 2 with the equi-dimensional model.

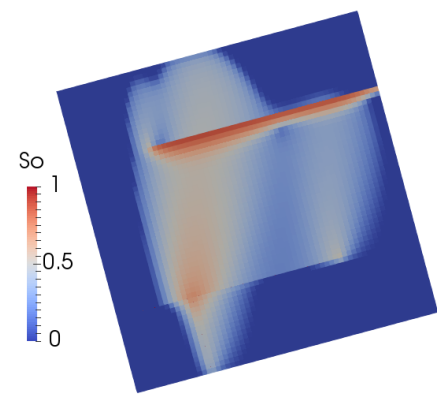

(a) Equi-dim.

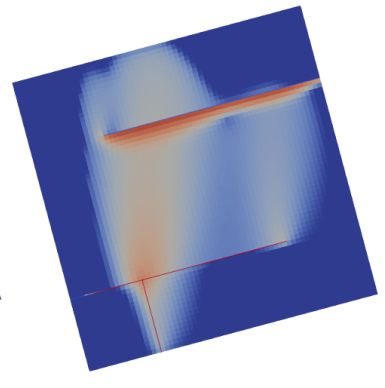

(b) $m f$ nonlinear

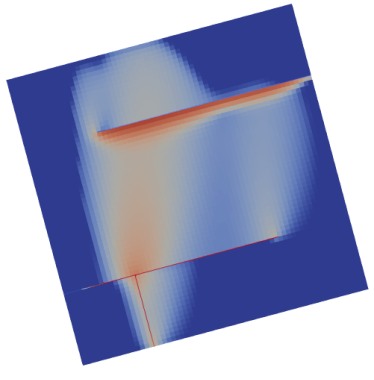

(c) $f$-upwind

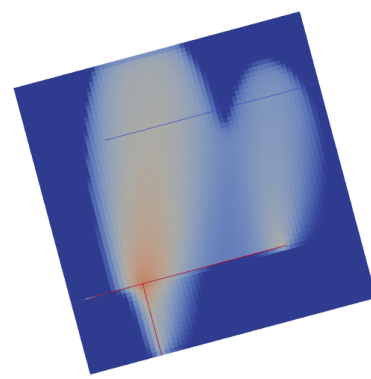

(d) $m$-upwind

Figure 11: From left to right, oil saturation at final time computed by the equi-dimensional and hybrid-dimensional models for the three drains and one barrier test case. 


\begin{tabular}{|c|c|c|c|c|c|c|c|}
\hline Model & Nb Cells & Nb dof & $\mathbf{N}_{\Delta t}$ & $\mathbf{N}_{\text {Newton }}$ & $\mathbf{N}_{\text {GMRes }}$ & $\mathbf{N}_{\text {Chop }}$ & $\mathbf{C P U [ s ]}$ \\
\hline equi-dimensional & 4896 & 4896 & 406 & 3.8 & 22 & 0 & 73 \\
\hline$m f$ nonlinear & 4096 & 4209 & 405 & 3.4 & 12 & 0 & 42 \\
\hline$f$ upwind & 4096 & 4209 & 403 & 3.5 & 16.4 & 0 & 47 \\
\hline$m$ upwind & 4096 & 4209 & 404 & 3.2 & 13.7 & 0 & 37 \\
\hline
\end{tabular}

Table 5: Numerical behavior of the equi-dimensional and hybrid-dimensional models for the 3 drains and 1 barrier test case. The mesh is Cartesian of size $68 \times 72$ for the equi-dimensional model and of size $64 \times 64$ for the hybrid-dimensional models.

\subsection{Desaturation by suction of a fractured porous medium}

We consider in this test case a water gas two-phase Darcy flow in the DFM model defined by the vertical domain $\Omega=(0,10 \mathrm{~m})^{2}$ and the fracture network exhibited in Figure 12. The matrix is homogeneous and charaterized by the isotropic permeability $\Lambda_{m}=1$ Darcy, the porosity $\phi_{m}=0.2$, the relative permeabilities $k_{r, m}^{g}\left(s^{g}\right)=\left(s^{g}\right)^{2}, k_{r, m}^{w}\left(s^{w}\right)=\left(s^{w}\right)^{3}$, and the capillary pressure $P_{c, m}\left(s^{g}\right)=-b_{m} \log \left(1-s^{g}\right)$ with $b_{m}=10^{6} \mathrm{~Pa}$. The fracture properties are set to $d_{f}=1 \mathrm{~mm}, \Lambda_{f}=\lambda_{f, n}=10^{4} \Lambda_{m}, \phi_{f}=0.35$, $k_{r, f}^{g}=k_{r, m}^{g}, k_{r, f}^{w}=k_{r, m}^{w}$, and $P_{c, f}\left(s^{g}\right)=-b_{f} \log \left(1-s^{g}\right)$ with $b_{f}=10^{4} \mathrm{~Pa}$. The fluid properties are defined by their dynamic viscosities $\mu^{g}=2.10^{-4}, \mu^{w}=10^{-3}$ Pa.s and their mass densities $\rho^{w}=1000$ and $\rho^{g}=1 \mathrm{Kg} \cdot \mathrm{m}^{-3}$.

The matrix and fracture domains are initially saturated by the water phase. The top boundary conditions are defined by the water saturation $s^{w}=1$ and the water pressure $p^{w}=10^{5} \mathrm{~Pa}$. The bottom boundary conditions are defined by the gas pressure $p^{g}=10^{5} \mathrm{~Pa}$ and the gas saturation fixed to $s^{g}=0.1$ in the matrix and to $s^{g}=P_{c, f}^{-1}\left(P_{c, m}(0.1)\right)$ in the fractures. The lateral boundaries are assumed impervious.

The simulation time is set to $t_{f}=10^{7} \mathrm{~s}$ which suffices to reach the stationary state for the three models. The time stepping is defined by the parameters $\Delta t^{1}=10 \mathrm{~s}, \Delta t^{\max }=10^{5} \mathrm{~s}$, and $\delta s^{o b j}=0.3$. The mesh is a uniform Cartesian grid of size $160 \times 160$.

Figures 12, 13, 14 exhibit the large discrepancies between the reference solution which is here given by the hybrid dimensional $m f$ nonlinear model and both $m f$ linear models. The $m f$ linear $f$ upwind model considerably overestimates the capillary barrier effect at the $m f$ interfaces due to bad approximation of the water mobility at the matrix-fracture interfaces for dry fractures. The $m f$ linear $m$ upwind model hardly sees the fractures in the sense that it does not capture the jumps of the water pressure and saturation at the matrix-fracture interfaces. The numerical behavior of the three models is similar as exhibited in Table 6 . 


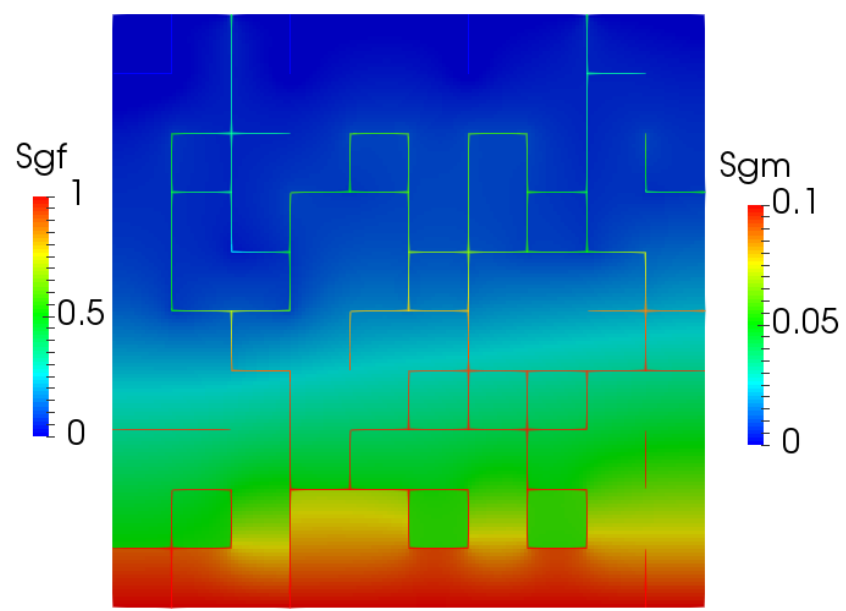

(a) $m f$ nonlinear

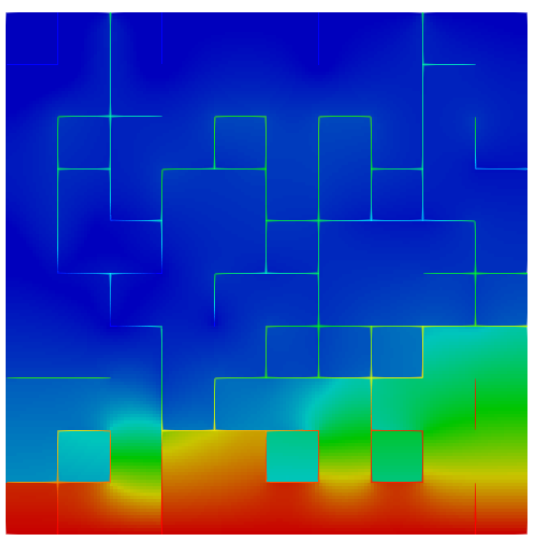

(b) $f$-upwind

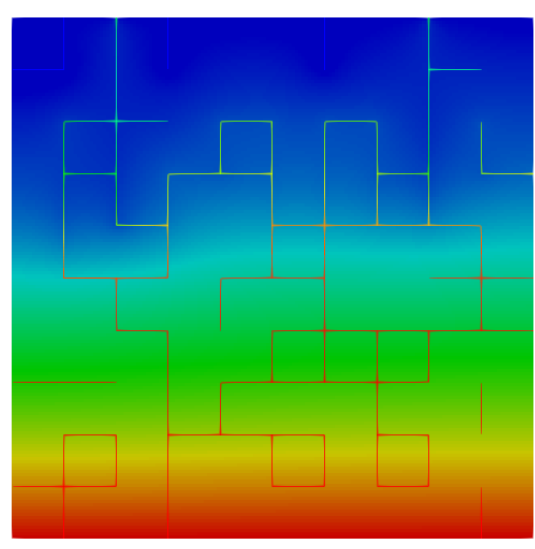

(c) m-upwind

Figure 12: Gas saturation at final time computed by the hybrid-dimensional models for the desaturation by suction test case.
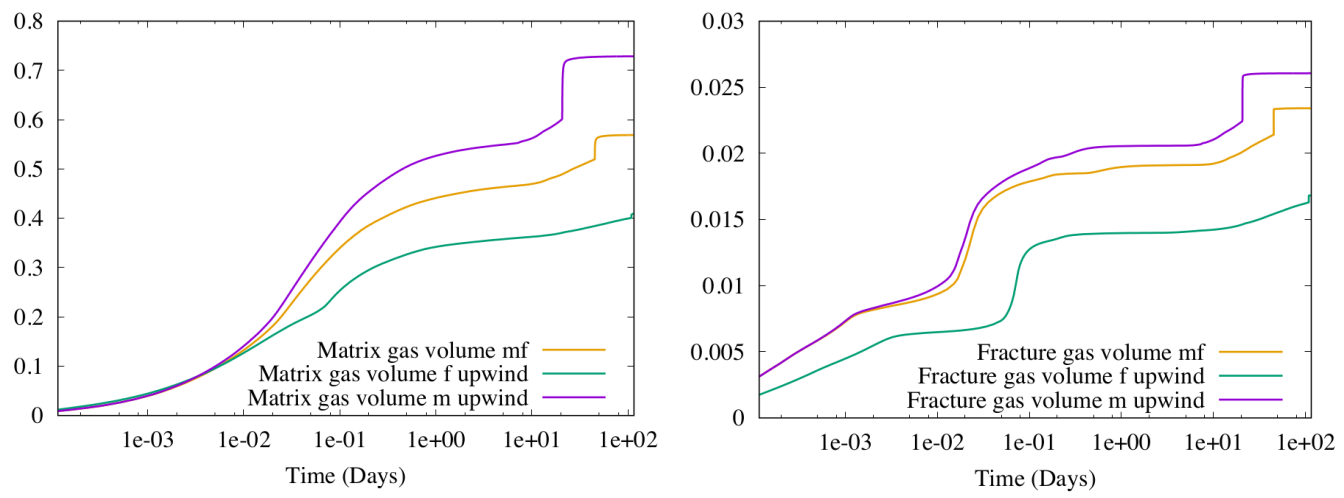

Figure 13: Gas volume in the matrix and in the fracture network as a function of time for the hybriddimensional models. 

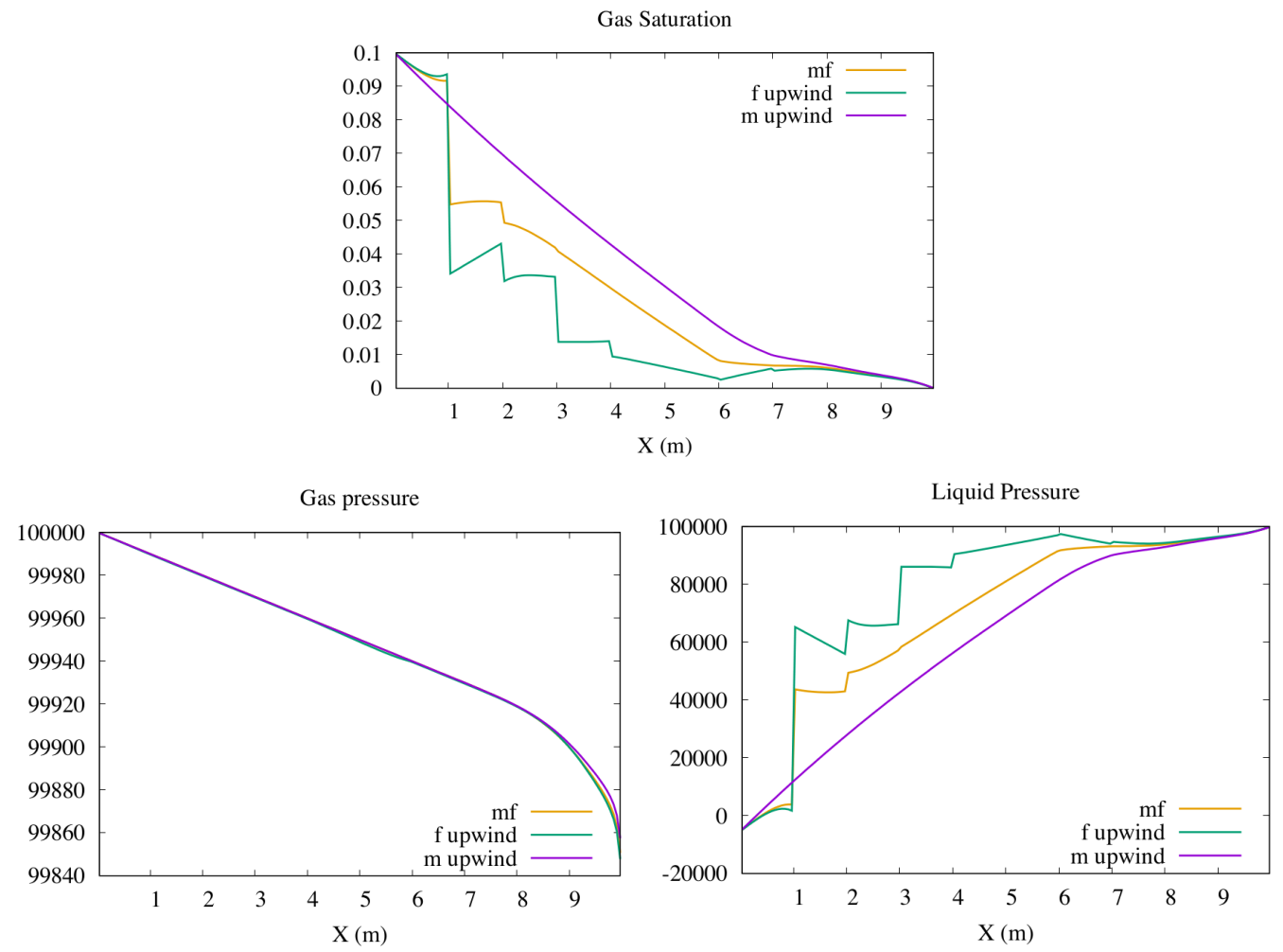

Figure 14: Cuts at $x=7.5 \mathrm{~m}$ of the gas saturation (top), water pressure (left bottom) and gas pressure (right bottom) at final simulation time for the for the hybrid-dimensional models.

\begin{tabular}{|l|c|c|c|c|c|c|c|}
\hline Model & Nb Cells & Nb dof & $\mathbf{N}_{\Delta t}$ & $\mathbf{N}_{\text {Newton }}$ & $\mathbf{N}_{\text {GMRes }}$ & $\mathbf{N}_{\text {Chop }}$ & CPU[s] \\
\hline$m f$ nonlinear & 25600 & 27117 & 227 & 11.2 & 105 & 20 & 2726 \\
\hline$f$ upwind & 25600 & 27117 & 199 & 10.9 & 111 & 15 & 2601 \\
\hline$m$ upwind & 25600 & 27117 & 245 & 10.8 & 92 & 24 & 2525 \\
\hline
\end{tabular}

Table 6: Numerical behavior of the hybrid-dimensional models for the desaturation test case. The mesh is Cartesian of size $160 \times 160$.

\section{$6 \quad 3 \mathrm{D}$ numerical experiments}

In this section, the numerical solutions of the hybrid-dimensional $m f$ nonlinear and $m f$ linear $f$ and $m$ upwind models are compared on a 3D numerical test case using a family of refined tetrahedral meshes. On such meshes, the Two-Point Flux Approximation of Section 3 is not consistent which motivates the use in this section of the alternative Vertex Approximate Gradient scheme described in [9]. This scheme is particularly well suited to tetrahedral meshes since it is based essentially on nodal unknowns. We refer to [9] for its detailed presentation for the two-phase hybrid-dimensional $m f$ nonlinear model. Let us remark that with such nodal scheme, the $m f$ interface unknowns are coupled along the connected fractures and hence cannot be eliminated. Since the test case considers highly permeable fractures, the $m f$ linear models can be further approximated using a continuous pressure model such as the one introduced in $[12,11]$. This continuous pressure model can be combined with either the $m$ upwinding of the mobilities as in $[12,11]$ or the more usual $f$ upwinding of the mobilities. The advantage of this continuous pressure approximation is to eliminate readily the interface unknowns for the $m f$ linear models with a negligible additional error for fractures acting as drains. 
The geometry of the domain and its coarsest tetrahedral mesh conforming to the fracture network are illustrated in Figure 15. The domain consists of a matrix domain of extension $100 \mathrm{~m} \times 100 \mathrm{~m} \times 100 \mathrm{~m}$ and of an homogeneous anisotropic permeability $\Lambda_{m}$, which is of 0.1 Darcy in $x$ and in $y$ directions and 0.01 Darcy in $z$ direction. The fracture network is assumed to be of constant aperture $d_{f}=1 \mathrm{~cm}$ and of isotropic permeability $\Lambda_{f}=\lambda_{f, n}=10$ Darcy. The matrix porosity $\phi_{m}$ is set to 0.2 and the fracture porosity $\phi_{f}$ to 0.4 . The matrix capillary pressure is given by $P_{c, m}\left(s^{o}\right)=-b_{m} \log \left(1-s^{o}\right)$, with $b_{m}=10^{4}$ $\mathrm{Pa}$, and the fracture capillary pressure by $P_{c, f}\left(s^{o}\right)=-b_{f} \log \left(1-s^{o}\right)$, with $b_{f}=100 \mathrm{~Pa}$. The matrix and the fracture network have the same relative permeabilities given by $k_{r, f}^{o}\left(s^{o}\right)=k_{r, m}^{o}\left(s^{o}\right)=\left(s^{o}\right)^{2}$ and $k_{r, f}^{w}\left(s^{w}\right)=k_{r, m}^{w}\left(s^{w}\right)=\left(s^{w}\right)^{2}$. The fluid properties are characterized by their dynamic viscosities $\mu^{o}=5.10^{-3}, \mu^{w}=10^{-3}$ Pa.s and by their mass densities $\rho^{w}=1000$ and $\rho^{o}=700 \mathrm{Kg} . \mathrm{m}^{-3}$.

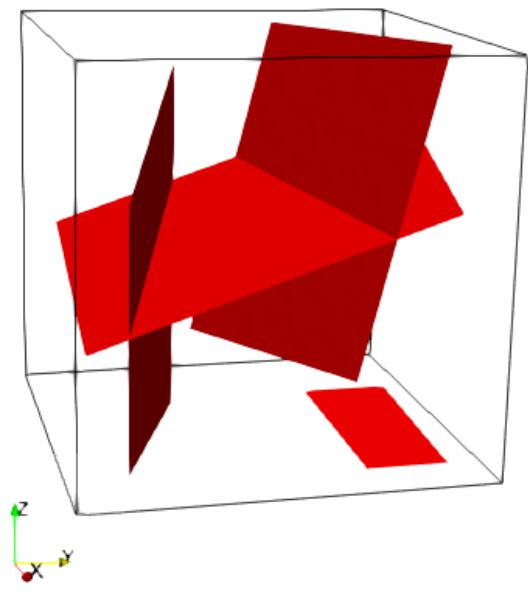

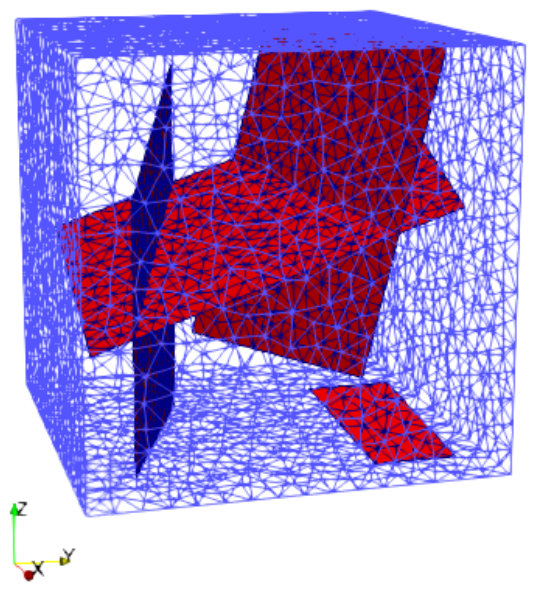

Figure 15: Geometry of the domain $\Omega=100 \mathrm{~m} \times 100 \mathrm{~m} \times 100$ $m$ with the fracture network in red (left), coarsest tetrahedral mesh with 47670 cells (right).

At initial time, the reservoir is fully saturated with water. Then, oil is injected from below, which is managed by imposing Dirichlet conditions at the bottom and at the top of the reservoir. We also impose $20.10^{5} \mathrm{~Pa}$ of overpressure w.r.t. the hydrostatic distribution of pressure. The oil then mounts by gravity, thanks to its lower density compared to water, and by the pressure gradient.

The sizes of the four tetrahedral meshes used for the simulations are reported in Table 7 together with the number of d.o.f. of the hybrid-dimensional $m f$ nonlinear and linear models. Note that the cell unknowns used for the VAG discretization are eliminated without any fill-in by static condensation after assembly of the Jacobian system at each Newton iteration resulting in a much lower number of d.o.f. compared with the number of cells. The number of d.o.f. for both $m f$ linear models is reduced compared with the number of d.o.f. of the $m f$ nonlinear model since the $m f$ interface d.o.f. are not included when using continuous pressure models. The parameters for the numerical resolution are as follows: crit $_{\text {Newton }}^{\text {rel }}=10^{-6}$, crit $_{\text {GMRes }}^{\text {rel }}=10^{-6}, \delta s^{o b j}=0.5$. The simulation of the flow covers the period of one year. The maximal time step size is $\Delta t_{\max }=0.1$ days, except for the $m f$ non linear model solved on the finest mesh, for which we set $\Delta t_{\max }=0.03$ days.

\begin{tabular}{|c|c|c|c|c|c|}
\hline Mesh & Nb Cells & Nb dof $(m f$ non lin. $)$ & Nb dof $(m f$ lin. $)$ & Nb dof el. $(m f$ non lin. $)$ & Nb dof el. $(m f$ lin. $)$ \\
\hline 1 & 47670 & 62763 & 57696 & 15093 & 9278 \\
\hline 2 & 124245 & 159744 & 148505 & 35499 & 23187 \\
\hline 3 & 253945 & 321670 & 301643 & 67725 & 46283 \\
\hline 4 & 452401 & 566243 & 535085 & 113842 & 80965 \\
\hline
\end{tabular}

Table 7: $\mathbf{N b}$ Cells is the number of cells of the mesh; $\mathbf{N b}$ dof is the number of d.o.f. (with two physical primary unknowns per d.o.f.); $\mathbf{N b}$ dof el. is the number of d.o.f. after elimination of cell unknowns without fill-in. 


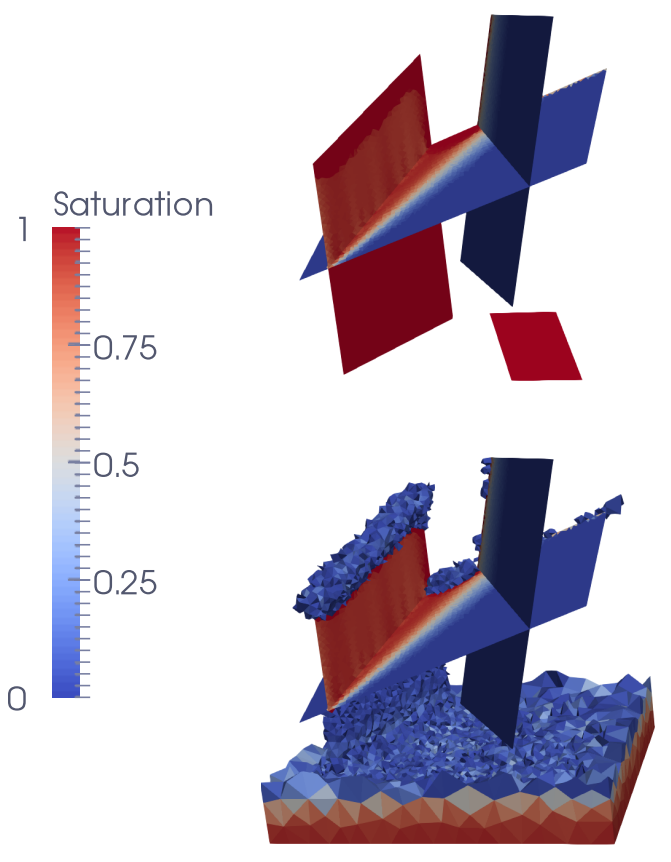

(a) $m f$ nonlinear
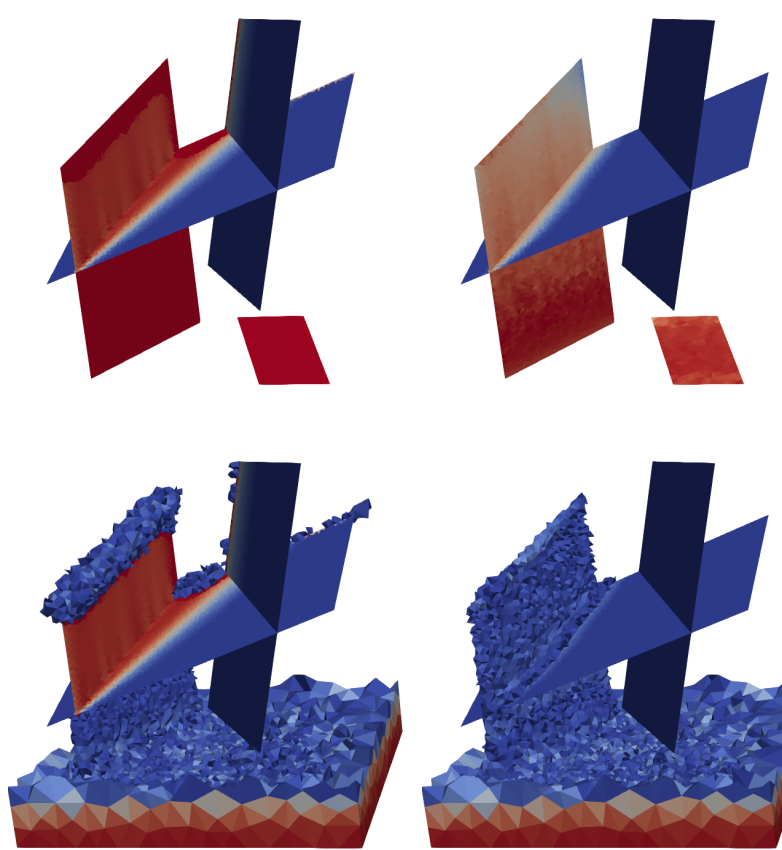

(b) $m$-upwind

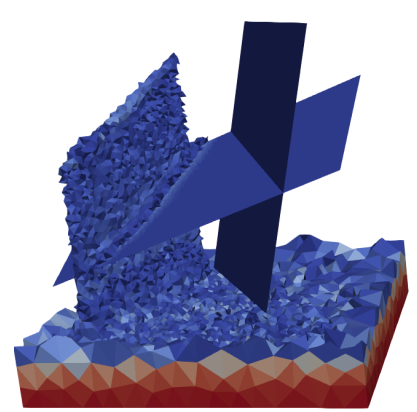

(c) f-upwind

Figure 16: Comparison of the oil saturation at time $t=295$ days for the hybrid-dimensional models using a matrix oil saturation threshold at 0.01 . Upper line: fracture network only.
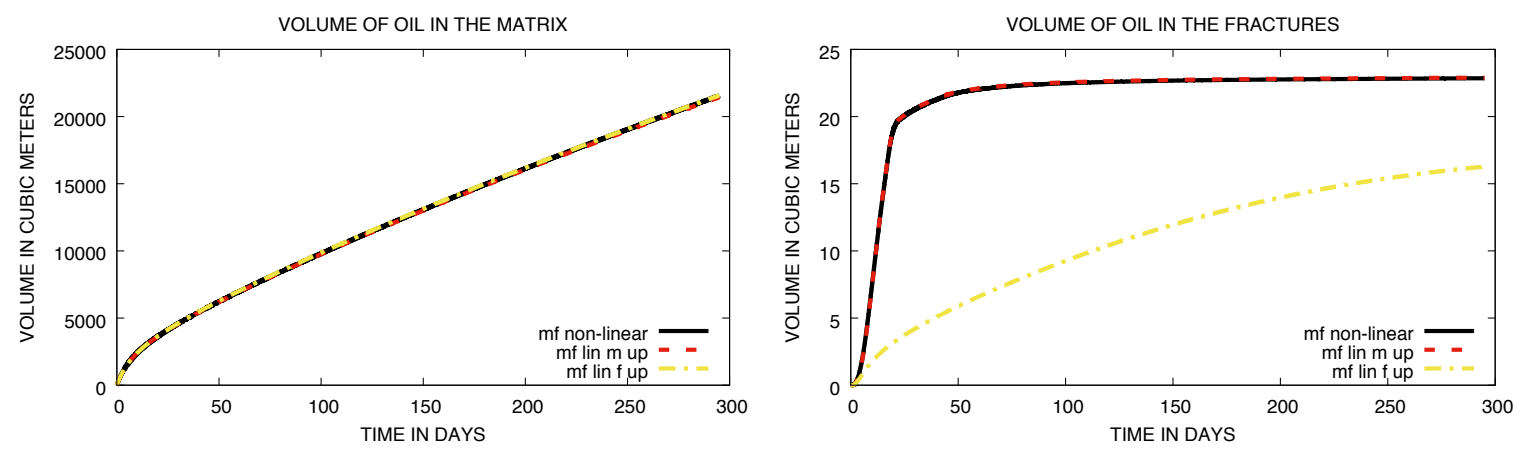

Figure 17: Comparison of the matrix and fracture volumes occupied by oil as a function of time for the different models. 

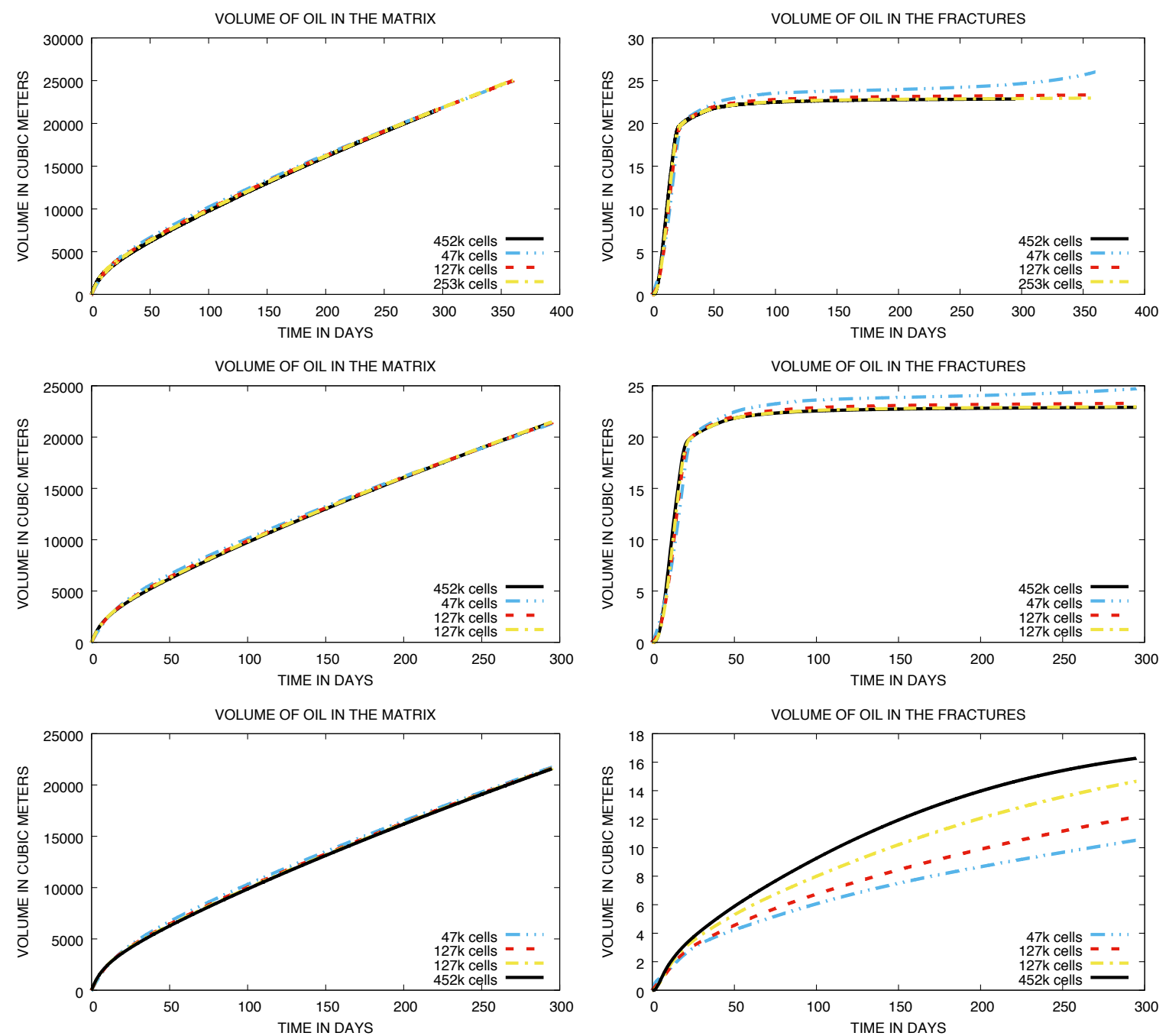

Figure 18: Comparison of the convergence for the different models: $m f$ non linear, $m f$ linear $m$ upwind and $m f$ linear $f$ upwind schemes (above to below). 


\begin{tabular}{|c|c|c|c|c|c|}
\hline Mesh & $\mathbf{N}_{\Delta t}$ & $\mathbf{N}_{\text {Newton }}$ & $\mathbf{N}_{\text {GMRes }}$ & $\mathbf{N}_{\text {Chop }}$ & CPU[s] \\
\hline mf non linear model \\
\hline 1 & 3103 & 9283 & 445677 & 6 & 14113 \\
\hline 2 & 3367 & 11846 & 869702 & 19 & 64326 \\
\hline 3 & 3890 & 16413 & 1365365 & 38 & 207589 \\
\hline 4 & 9947 & 32625 & 2792423 & 29 & 775567 \\
\hline mf linear $m$ upwind model \\
\hline 1 & 3075 & 3655 & 202178 & 0 & 3042 \\
\hline 2 & 3075 & 3781 & 282025 & 0 & 10325 \\
\hline 3 & 3075 & 4122 & 387035 & 0 & 27839 \\
\hline 4 & 3075 & 4737 & 536641 & 0 & 63766 \\
\hline$m f$ linear $f$ upwind model \\
\hline 1 & 3075 & 3621 & 199874 & 0 & 2879 \\
\hline 2 & 3075 & 3782 & 283473 & 0 & 9943 \\
\hline 3 & 3075 & 4125 & 388904 & 0 & 26917 \\
\hline 4 & 3075 & 4703 & 538231 & 0 & 63700 \\
\hline
\end{tabular}

Table 8: Mesh refers to the meshes defined in Table 7; $\mathbf{N}_{\Delta t}$ is the number of successful time steps; $\mathbf{N}_{\text {Newton }}$ is the total number of Newton iterations (for successful time steps); $\mathbf{N}_{\text {Chop }}$ is the number of time step chops.

This test shows the discrepancy between the $m f$ linear $f$ upwind model and the two other hybriddimensional models for a simulation period of fracture filling time scale. As one would expect, Figures 17 and 18 reveal that the $m f$ linear $f$ upwind model overestimates the exchange of oil from the fracture network into the matrix, which leads on the one hand to a higher prediction of oil volume in the global domain and on the other hand to a slower filling of the fracture network. This is basically due to the fact that the flux going out of the fractures does not take into account the saturation jump at the $m f$ interfaces for the $m f$ linear $f$ upwind model in contrast with the $m f$ nonlinear and the $m f$ linear $m$ upwind models. Also, from Figure 18 the solution of the $m f$ linear $f$ upwind model is strongly mesh dependent. For the $m f$ linear $m$ upwind model and the $m f$ nonlinear model, we observe a much better convergence in space. Table 8 exhibits a large computational overhead for the $m f$ nonlinear model compared with the $m f$ linear models especially on fine meshes. Such a large computational overhead was not observed on the 2D test cases using the TPFA discretization, probably thanks to the nonlinear and linear eliminations of the $m f$ interface unknowns. Such eliminations cannot be performed for the VAG discretization due to the coupling of the node unknowns along the connected fractures. On the other hand, for this test case, the $m f$ nonlinear model on the coarsest mesh is more accurate than the $m f$ linear $f$ upwind model on the finest mesh.

\section{Conclusion}

The numerical experiments exhibit on various test cases a better accuracy of the hybrid-dimensional $m f$ nonlinear model based on nonlinear transmission conditions compared with the hybrid-dimensional models based on linear transmission conditions.

The hybrid-dimensional $m f$ linear $m$ upwind model, which matches basically with the continuous phase pressures model $[7,25,23,18,12,11]$ in the case of fractures acting as drains, is shown to fail to produce a good approximation of the equi-dimensional model once the fractures are filled with the non-wetting phase. This is a modeling error due to an overestimation of the capillary pressure inside the filled fractures. This situation is quite different to what happens for single phase flows for which continuous and discontinuous pressure models basically match as soon as the ratio between the fracture normal permeability and the fracture width is large compared with the ratio between 
the matrix permeability and its characteristic length. As expected, for fractures acting as capillary barriers, the hybrid-dimensional $m f$ linear $m$ upwind model also fails to reproduce the capillary barrier effect and only accounts for permeability barriers.

The hybrid-dimensional $m f$ linear $f$ upwind model tends to overestimate the non-wetting phase flux going out of the permeable fractures since it does not capture the saturation jump at $m f$ interfaces in contrast with the $m f$ nonlinear and $m f$ linear $m$ upwind models. Both $m f$ linear $f$ and $m$ upwind models fail to reproduce an accurate solution for the desaturation by suction test case. The $f$ upwind model overestimates the capillary barrier effect at $m f$ interfaces while the $m$ upwind model does not capture the saturation and water pressure jumps at dry fracture interfaces.

None of the $m f$ linear models are able to take into account the gravity segregation in the fracture width which can have a strong impact for small capillary pressures or for large apertures such as in the case of faults.

A moderate computational overhead of the $m f$ nonlinear model compared with the $m f$ linear models is obtained in the 2D simulations using the TPFA scheme thanks to the elimination of the $m f$ interface unknowns at both the linear and nonlinear levels. The VAG scheme does not allow such eliminations for the $m f$ nonlinear model due to the coupling of the nodes along the connected fractures. It results that the $m f$ nonlinear model combined with the VAG discretization exhibits a large computational overhead compared with the continuous pressure model. To extend the elimination of the $m f$ interface unknowns to consistent discretizations on general meshes, a promising approach developped in [1] is to use face-based discretizations [8] rather than nodal-based discretizations.

\section{References}

[1] J. Aghili, K. Brenner, J. Hennicker, R. Masson, L. Trenty, Hybrid Finite Volume discretization of two-phase Discrete Fracture Matrix models with nonlinear interface solver, ECMOR XVI 16th European Conference on the Mathematics of Oil Recovery, Sep 2018, Barcelona, France, doi $10.3997 / 2214-4609.201802272$

[2] Alboin, C., Jaffré, J., Roberts, J., Serres, C.: Modeling fractures as interfaces for flow and transport in porous media. Fluid flow and transport in porous media 295, 13-24, 2002.

[3] Ahmed, E., M., Jaffré, J., Roberts, J. E., A reduced fracture model for two-phase flow with different rock types, Mathematics and Computers in Simulation, 7, pp. 49-70, 2017.

[4] Ahmed, R., Edwards, M.G., Lamine, S., Huisman, B.A.H., Control-volume distributed multi-point flux approximation coupled with a lower-dimensional fracture model, J. Comp. Physics, 462-489, Vol. 284, 2015.

[5] Angot, P., Boyer, F., Hubert, F. Asymptotic and numerical modeling of flows in fractured porous media, M2AN, 43,2, pp; 239-275, 2009.

[6] R.G. Bentsen and J. Anli. Using parameter estimation techniques to convert centrifuge data into a capillary-pressure curve, SPE Journal, 17(1):57-64, 1977.

[7] Bogdanov, I., Mourzenko, V., Thovert, J.-F., Adler, P. M., Two-phase flow through fractured porous media, Physical Review E 68, 026703, 2003.

[8] K. Brenner; J. Hennicker; R. Masson; P. Samier. Gradient discretization of hybrid-dimensional Darcy flow in fractured porous media with discontinuous pressures at matrix-fracture interfaces, IMA Journal of Numerical Analysis, 37,3, pp. 1551-1585, 2016.

[9] K. Brenner, J. Hennicker, R. Masson, P. Samier. Hybrid-dimensional modeling of two-phase flow through fractured porous media with enhanced matrix fracture transmission conditions. J. Comp. Physics, 100-124, Vol. 357, 2018. 
[10] Brenner, K., Groza, M., Guichard, C., Lebeau, G. and Masson, R. Gradient discretization of Hybrid-Dimensional Darcy Flows in Fractured Porous Media. Numerische Mathematik, 134,3, pp. 569-609, 2016.

[11] Brenner, K., Groza, M., Jeannin, L., Masson, R., Pellerin, J. Immiscible two-phase Darcy flow model accounting for vanishing and discontinuous capillary pressures: application to the flow in fractured porous media, Computational Geosciences, online july 2017.

[12] Brenner, K., Groza, M., Guichard, C., Masson, R. Vertex Approximate Gradient Scheme for Hybrid-Dimensional Two-Phase Darcy Flows in Fractured Porous Media. ESAIM Mathematical Modeling and Numerical Analysis, 49, pp. 303-330, 2015.

[13] R. H. Brooks and A. T. Corey. Hydraulic properties of porous media and their relation to drainage design. Transactions of the ASAE, 7(1):0026-0028, 1964.

[14] J. Droniou, J. Hennicker, and R. Masson. Numerical analysis of a two-phase flow discrete fracture model, Numerische Mathematik, doi: 10.1007/s00211-018-0994-y, online 5 september 2018.

[15] R. Eymard, T. Gallouët, C. Guichard, R. Herbin, and R. Masson. TP or not TP, that is the question. Comput. Geosci., 18:285-296, 2014.

[16] Flauraud, E., Nataf, F., Faille, I., Masson, R. Domain Decomposition for an asymptotic geological fault modeling, Comptes Rendus à l'Académie des Sciences, Mécanique, 331, pp 849-855, 2003.

[17] Flemisch B., Berre I., Boon W., Fumagalli A., Schwenck N., Scotti A., Stefansson I., Tatomir A. Benchmarks for single-phase flow in fractured porous media, Advances in Water Resources 239-258, Vol. 111, 2018.

[18] Hoteit, H., Firoozabadi, A. Numerical modeling of two-phase flow in heterogeneous permeable media with different capillarity pressures. Advanced Water Resources 31, pp. 56-73, 2008.

[19] Jaffré, J., Martin, V., Roberts, J. E. Modeling fractures and barriers as interfaces for flow in porous media, SIAM J. Sci. Comput. 26,5, pp. 1667-1691, 2005.

[20] Jaffré, J., Mnejja, M., Roberts, J. E., A discrete fracture model for two-phase flow with matrixfracture interaction, Procedia Computer Science 4, pp. 967-973 (2011)

[21] Karimi-Fard, M., Durlovski, L.J., Aziz, K. An efficient discrete-fracture model applicable for general-purpose reservoir simulators, SPE journal, june 2004.

[22] Lacroix, S., Vassilevski, Y. V., Wheeler, M. F.: Decoupling preconditioners in the implicit parallel accurate reservoir simulator (IPARS). Numerical Linear Algebra with Applications, 8, 537-549 (2001)

[23] Monteagudo, J.E.P. and Firoozabadi, A. Control-Volume Model for Simulation of Water Injection in Fractured Media: Incorporating Matrix Heterogeneity and Reservoir Wettability Effects, SPE Journal 12, 3, 2007.

[24] Mualem, Y. A new model for predicting the hydraulic conductivity of unsaturated porous media, Water Resour. Res., 12, 513-522, 1976

[25] Reichenberger, V., Jakobs, H., Bastian, P., Helmig, R.: A mixed-dimensional finite volume method for multiphase flow in fractured porous media. Adv. Water Resources 29, 7, pp. 10201036, 2006.

[26] Scheichl, R., Masson, R., Wendebourg, J.: Decoupling and block preconditioning for sedimentary basin simulations. Computational Geosciences, 7, 295-318 (2003) 
[27] Sandve, T.H., Berre, I., Nordbotten, J.M. An efficient multi-point flux approximation method for Discrete Fracture-Matrix simulations, JCP 231 pp. 3784-3800, 2012.

[28] Tunc, X., Faille, I., Gallouët, T., Cacas, M.C., Havé, P. A model for conductive faults with non matching grids, Comp. Geosciences, 16, pp. 277-296, 2012.

[29] Van Genuchten, M.T. A Closed-form Equation for Predicting the Hydraulic Conductivity of Unsaturated Soils, Soil Sci. Soc. Am. J. 1980, 44, 892-898. 